Elsevier Editorial System(tm) for Neurolmage

Manuscript Draft

Manuscript Number: NIMG-05-251R2

Title: Change detection in children with autism: an auditory event-related fMRI study.

Article Type: Regular Article

Section/Category:

Keywords:

Corresponding Author: Dr. Marie Gomot, PhD

Corresponding Author's Institution: INSERM U619

First Author: Marie Gomot, PhD

Order of Authors: Marie Gomot, PhD; Frederic A Bernard, PhD; Matthew H Davis, PhD; Matthew K Belmonte, PhD; Chris Ashwin, PhD; Edward T Bullmore, Pr.; Simon Baron-Cohen, Pr.

Manuscript Region of Origin:

Abstract: Autism involves impairments in communication and social interaction, as well as high levels of repetitive, stereotypic and ritualistic behaviours, and extreme resistance to change. This latter dimension, whilst required for a diagnosis, has received less research attention. We hypothesise that this extreme resistance to change in autism is rooted in atypical processing of unexpected stimuli. We tested this using auditory event-related fMRI to determine regional brain activity associated with passive detection of infrequently occurring frequency-deviant and complex novel sounds in a no-task condition. Participants were twelve 10 to 15-year-old children with autism, and a group of 12 age- and sex-matched healthy controls. During deviance-detection, significant activation common to both groups was located in the superior temporal and inferior frontal gyri. During 'novelty-detection', both groups showed activity in the superior temporal gyrus, the temporo-parietal junction, the superior and inferior frontal gyri and the cingulate gyrus. 
Children with autism showed reduced activation of the left anterior cingulate cortex during both deviance and novelty detection. During 'novelty-detection' children with autism also showed reduced activation in the bilateral temporo-parietal region, and in the right inferior and middle frontal areas. This study confirms previous evidence from ERP studies of atypical brain function related to automatic change detection in autism. Abnormalities involved a cortical network known to have a role in attention-switching and attentional resource distribution. These results throw light on the neurophysiological processes underlying autistic 'resistance to change'. 
Unité de Recherche U619

Dynamique et pathologie du

développement cérébral

\section{Inserm}

Institut national

de la santé et de la recherche médicale

Marie GOMOT, PhD

INSERM U 619

Child Psychiatry Unit- CHU Bretonneau

2, Boule vard Tonnellé

37044 - Tours Cedex

FRANCE

Tours, July the $7^{\text {th }}, 2005$

\section{Change detection in children with autism: an auditory event-related fMRI study}

Marie Gomot et al: NIMG-05-251

Dear Drs Toga and Fletcher,

Thank you for inviting us to resubmit this manuscript to Neurolmage.

We are grateful to the reviewers for the ir constructive comments. Especially we would like to thanks reviewer 2 who did insightful criticisms. We did take into account most of the remarks and we feel that the paper is improved as a result. Reviewer 2's observations allowed getting a better definition of the terms and concepts used. We corrected the text according to these criticisms, but we think that some of the modifications required regarding fundamental concepts on deviance processing would not serve the manuscript. We didn't wish for adding to many additional details about these fundamental concepts, in order to keep focus on the main aim of the study which was to study change processing in an autistic population. Moreover, we felt a bit disconcerted by some of the comments (See comments $5,7,19,21,24,30$ and 34 for example).

We look forward to hearing from you about the acceptability of the revised manuscript for publication in Neurolmage.

Yours Sincerely,

\section{Dr. Marie Gomot}


Draft 06.07.05

\title{
Change detection in children with autism: an auditory event-related fMRI study
}

\author{
Marie Gomot ${ }^{12}$, Frederic A. Bernard ${ }^{3}$, Matthew H. Davis ${ }^{4}$, Matthew K. Belmonte ${ }^{1}$, \\ Chris Ashwin ${ }^{1}$, Edward T. Bullmore ${ }^{3}$, Simon Baron-Cohen ${ }^{1}$
}
${ }^{1}$ Autism Research Centre, Department of Psychiatry, University of Cambridge, UK.
${ }^{2}$ INSERM U619; Université François Rabelais; CHRU Bretonneau; Tours, France.
${ }^{3}$ Brain Mapping Unit and Wolfson Brain Imaging Centre, University of Cambridge; Addenbrooke's Hospital, UK.
${ }^{4}$ MRC Cognition and Brain Sciences Unit, Cambridge, UK.

\author{
Corresponding author: \\ Dr Marie Gomot \\ INSERM U619 \\ Service d'Exp lorations Fonctionnelles en Pédopsychiatrie \\ 2 bd Tonnellé \\ 37044 Tours Cedex \\ France \\ E-mail: m.gomot@,chu-tours.fr \\ Tel: 0033247478664 \\ Fax: 0033247473846
}

Total number of words: 5137

Running title: Change detection in autism 


\section{SUMMARY:}

Autism involves impairments in communication and social interaction, as well as high levels of repetitive, stereotypic and ritualistic behaviours, and extreme resistance to change. This latter dimension, whilst required for a diagnosis, has received less research attention. We hypothesise that this extreme resistance to change in autism is rooted in atypical processing of unexpected stimuli. We tested this using auditory event-related fMRI to determine regional brain activity associated with passive detection of infrequently occurring frequency-deviant and complex novel sounds in a no-task condition. Participants were twelve 10 to 15 -year-old children with autism, and a group of 12 age- and sex-matched healthy controls. During deviance-detection, significant activation common to both groups was located in the superior temporal and inferior frontal gyri. During 'novelty-detection', both groups showed activity in the superior temporal gyrus, the temporo-parietal junction, the superior and inferior frontal gyri and the cingulate gyrus. Children with autism showed reduced activation of the left anterior cingulate cortex during both deviance and novelty detection. During noveltydetection children with autism also showed reduced activation in the bilateral temporoparietal region, and in the right inferior and middle frontal areas. This study confirms previous evidence from ERP studies of atypical brain function related to automatic change detection in autism. Abnormalities involved a cortical network known to have a role in attention-switching and attentional resource distribution. These results throw light on the neurophysiological processes underlying autistic 'resistance to change'.

Keywords: Autism - fMRI - Deviance-detection - Novelty-detection - Auditory - Oddball Anterior cingulate cortex - Neuroimaging. 


\section{INTRODUCTION}

The ability to detect unexpected new events is an essential part of normal adaptive behaviour which is critical in rapidly changing environments, particularly social situations. Autism is a neurodevelopmental condition in which change processing may be atypical, possibly set at a too sensitive level. Autism involves significant social and communication difficulties, and this has received considerable research attention. A key characteristic of autism that may arise from atypical processing of irregular events is sometimes referred to as 'resistance to change'. This was initially described as a 'need for sameness' (Kanner, 1943). It may be expressed in terms of tantrums as a response to change, or in terms of restricted interests and repetitive or stereotyped behaviours, and is present at all ages (Kobayashi and Murata, 1998).

Resistance to change may also occur at the sensory level; individuals with autism - especially infants - display unusual behaviours in response to changes in sensory stimuli (Rogers et al., 2003; Malvy et al., 2004). This is especially the case in the auditory modality, where paradoxical responses to sounds, both hyper- and hypo-reactivity to noises, are reported (Wing, 2003). Finally, when left to their own devices, children with classic autism tend to compulsively create patterns, which may reflect a strong desire to impose control over changing events. (Frith, 1970). Taken together, these clinical features suggest that people with autism may have a basic difficulty in automatically orientating to changing sensory stimuli. Although resistance to change is a main feature of autism, the brain processes underlying this aspect of the condition remain poorly understood.

The neural correlates of change detection are commonly studied through the oddball paradigm. This involves embedding infrequent acoustic changes in a sequence of repetitive ('standard') stimuli. The infrequent stimuli are either slightly deviant from the standard (for 
example, tones of a higher pitch) or are highly deviant unique stimuli (unfamiliar sounds, designed to capture attention). In the oddball paradigm, the slightly deviant stimuli are referred to as simply 'deviant', and the highly deviant unique stimuli are referred to as 'novel' and evoke distinct patterns of brain activation. These brain processes underlying pre-attentive auditory change detection have been investigated in typical populations largely using electrophysiological methods, particularly event-related potentials (ERPs).

The ERP response evoked by any deviant stimuli is called Mismatch Negativity (MMN). It is assumed to reflect a pre-attentional change detection process and its recording does not require active participation from the subject. $\mathrm{MMN}$ is thought to reflect the automatic detection of a difference between the active sensory memory trace of the recent repetitive event (the standard) and an incoming deviant stimulus (Näätänen, 1992; Näätänen et al., 2005). Several studies using different methods have identified cortical generators of MMN (Picton et al. 2000). The main MMN component is generated bilaterally in the supratemporal part of the auditory cortex and might be associated with acoustic change detection (see Alho, 1995 for review). An additional generator has been identified in the frontal region (Giard et al., 1990; Alho et al., 1994, Alain et al., 1998) which may be related to initiation of an involuntary attention switch toward the detected change (Giard et al., 1990; Näätänen, 1992; Schröger, 1996). MMN can be recorded early in development and mechanisms underlying this response are assumed to be similar across the lifespan (Cheour-Luhtanen et al., 1996; Gomot et al., 2000).

A few studies on auditory change detection mechanism underlying MMN have been conducted with people with autism, and these have yielded contrad ictory findings. This is probably due to the variability in the populations studied and in the nature of the stimuli used. In response to tones, MMN amplitude has been found to be normal (Kemner et al., 1995; Čeponienè et al., 
2003), reduced (Seri et al., 1999) or larger (Ferri et al., 2003) in autism than in controls. In order to understand the underlying neurophysiological processes, Gomot et al. (2002) used scalp potential and scalp current density mapping to study MMN in children with autism. Results showed that MMN topography in autism was characterised by atypical electrical activity (additional positive currents) recorded over left frontal sites. Although the precise location of the brain mechanisms underlying the abnormalities in the scalp-recorded ERPs remains to be clarified, this result suggests that cortical or subcortical sources located in the anterior part of the left hemisphere could be involved in the atypical processing of change in children with autism.

In addition to MMN, the ERP response typically elicited by any novel stimulus occurring in a sequence of repetitive sounds is the P3a or Novelty P3. This response is thought to be associated with involuntary switching of attention toward stimulus changes occurring outside the current focus of attention (Schröger, 1996; Escera et al., 1998, 2000). Several brain areas are assumed to participate in P3a generation. These include the dorsolateral prefrontal cortex, the temporo-parietal junction, the posterior hippocampal region (Knight, 1996) and the auditory cortex (Alho et al., 1998). Most studies carried out in autism on electrical responses associated with novelty processing have involved active novel-target detection. Results showed that the response is significantly smaller in children with autism compared to controls (Courchesne et al., 1984, 1985; Lincoln et al., 1993; Kemner et al. 1995). P3a in response to novel speech stimuli in a no-task condition has been shown to be reduced in autism (Čeponienè et al., 2003). These findings have been interpreted as reflecting an atypical orientation to novel stimuli (Bomba and Pang, 2004).

Despite excellent temporal resolution, electrophysiological methods do not allow the precise localisation of the brain regions involved in atypical change detection in people with autism. fMRI offers the potential to identify the sub-cortical and cortical anatomical structures in 
which these effects occur. Automatic auditory change-detection has recently been studied in typical adults using fMRI (Rinne et al., 2005, Molholm et al., 2005). Despite the contamination of the stimuli by the acoustic noise of fMRI, it is possible to carry out passive MMN-like paradigms, as long as the physical features of the auditory stimuli differ sufficiently to allow detection (Opitz et al., 2002). Moreover, although fMRI noise alters sensory response in auditory cortex, it does not affect the change-detection (MMN) response (Novitski et al., 2001). Various block design fMRI studies have been carried out to assess change detection using the classical passive oddball paradigm (Celsis et al., 1999; Opitz et al., 1999a; Liebenthal et al., 2003; Jääskeläinen et al., 2004; Sabri et al., 2004) or paradigms using multimodal transitions as the deviant event (Downar et al. 2000) or the noise produced by the scanner itself during gradient switching as the deviant stimulus (Mathiak et al., 2002). Most studies report bilateral activation of the superior temporal gyrus in response to the deviant stimuli. A few have highlighted the activation of the other brain regions which are known to be involved in the processing of infrequent sound changes. Using event-related fMRI, Opitz et al. (2002) and Doeller et al. (2003) found additional activation in the inferior frontal gyrus in response to deviant stimuli. Thus, even though the choice of the design used is crucial to capture deviance-related brain activity outside the auditory cortex (Schall et al., 2003), fMRI has proven to be a useful tool to investigate the cerebral basis of deviance-detection.

Imaging of novelty processing has not received much research attention. Some fMRI studies have addresses the brain regions associated with novelty detection in a no-task condition, and these have only been conducted with typical volunteers (Opitz et al., 1999b; Downar et al., 2002). These studies found bilateral activation of the superior temporal gyrus. The latter study also revealed activation of a common network across modalities involving the temporo-parietal junction, the inferior frontal region, the cingulate gyrus and the insula. 
The aims of the current study were (1) to test the hypothesis of atypical brain activation during automatic acoustic change detection in children with autism; and (2) to determine if these activations differ according to the novelty of the stimuli. We used event-related fMRI to study the processing of unattended frequency-deviant and complex novel sounds and to highlight the brain regions underpinning these processes in children with and without autism. We predicted that in autism we would observe a perturbation of the cerebral network involved in involuntary attention switching during auditory change detection, including both the frontal and possibly the temporo-parietal region.

\section{METHOD}

\section{Participants}

Twelve male children with high-functioning autism (HFA) and twelve typically developing children matched for sex, age, and IQ participated in the experiment. Children were aged 10 to 15 years (mean $\pm \mathrm{SD}$ : autism $13.5 \pm 1.6$; control $13.8 \pm 1$ ) and were all right-handed. Participants with autism were diagnosed according to the DSM-IV-R criteria (APA, 2000) and the ADI-R (Lord et al., 1994). Mean full-scale IQ scores based on the WASI (Wechsler Abbreviated Scale of Intelligence, 1999) did not differ between the autism group and the control group and were $116 \pm 18$ and $120 \pm 7$ respectively.

Participants with hearing abnormality (as assessed by a subjective audiometric task), infectious, metabolic or neurological disorders, or contraindication for MRI were excluded. No child was on medication at the time of the scanning session and all patients were free of psychotropic drugs for a period of at least two months before the study. The study was approved by the Addenbrooke's NHS Trust Local Research Ethics Committee. Written 
informed consent was obtained from the parents or guardians of the subjects, according to the Declaration of Helsinki, and from the children themselves.

\section{Auditory stimuli and experimental paradigm}

Change-detection processes were studied through an oddball paradigm with three different types of stimuli, with an event-related fMRI design. Auditory sequences including 'Standard' (probability of occurrence: $p=0.82)$, 'Deviant' $(p=0.09)$ and 'Novel' sounds $(p=0.09)$ were delivered binaurally through non-ferromagnetic headphones. The Standard repetitive stimulus was a three-partial sound composed of 3 frequencies (sinusoids: $500 \mathrm{~Hz}, 1000 \mathrm{~Hz}, 1500 \mathrm{~Hz}$ ) with the second and third components 3 and $6 \mathrm{~dB}$ respectively lower in intensity than the first component. The Deviant sound differed from the Standard by a $30 \%$ change in frequency of all partial components (components: $650 \mathrm{~Hz}, 1300 \mathrm{~Hz}$ and $1950 \mathrm{~Hz}$ ). The Standard and Deviant sounds were similar to those used in previous ERP and fMRI studies of deviance processing (Tervaniemi et al., 2000; Opitz et a1., 2002). The Novel sounds differed from the Standard sounds with regard to their frequency composition, basic frequencies, and frequency transitions. They were originally sampled from a musician's keyboard and digitally prepared for stimulation purposes using the CoolEdit $\subseteq$ sound editor. The resulting stimuli were always novel non-identifiable complex sounds, each of them comprising a different spectrum of frequency composition. These novel sounds were kindly provided by B. Müller (Müller et al., 2002). All sounds had an overall intensity level of $85 \mathrm{~dB}$ SPL and a duration of $80 \mathrm{~ms}$ (including $10 \mathrm{~ms}$ rise/fall). Stimuli were presented with a constant stimulus onset asynchrony (SOA) of $625 \mathrm{~ms}$, in pseudo-random order with the following constraints: the five first stimuli

were Standard sounds, each Deviant or Novel stimulus was preceded by at least 3 Standard sounds, and the last five stimuli were Standard sounds. The minimum time interval between two Deviant or Novel stimuli was thus $2500 \mathrm{~ms}$. The total number of stimuli was 668, 
including 58 Deviants and 58 Novels. In order to control for tonotopic effect, the Deviant sound was swapped with the Standard sound halfway through the sequence. During the acquisition sequence subjects were instructed to watch a video of bird migration. Six 'Resting' periods of 10 seconds each (involving video watching only with no auditory stimuli) were interspersed, including one at the beginning and one at the end of the auditory sequence.

\section{fMRI procedure}

\section{Data acquisition}

Magnetic resonance data were acquired on a $3 \mathrm{~T}$ whole body system consisting of a Bruker Medspec 30/100 spectrometer (Ettlingen, Germany) and a $910 \mathrm{~mm}$ bore whole body actively shielded magnet (Oxford Magnet Technology, Oxford, UK). Functional images were collected using $\mathrm{T} 2 *$-weighted gradient-echo echo-planar imaging $(\mathrm{EPI})$ with $\mathrm{TR}=2.5 \mathrm{~s}, \mathrm{TE}=$ $27.5 \mathrm{~ms}$, flip angle $=82^{\circ}$. The acquisition volume consisted of 20 oblique-axial slices with slice thickness $=4 \mathrm{~mm}$ and interslice gap $=1 \mathrm{~mm}$. The matrix was $64 \mathrm{x} 64$ with a $20 \mathrm{~cm}$ field of view, yielding in an in-plane resolution of $3.125 \times 3.125 \mathrm{~mm}$. Two hundred volumes were acquired for each participant. The first five volumes acquired were discarded to allow for

signal equilibration, giving a total of 195 volumes used in the analysis. In order to minimise the contamination of the auditory stimuli by the scanner noise, each stimulus was delivered exactly at the time when the scanner was the quietest, i.e., in the temporal gap between two slices. Hence stimulus delivery was clo sely syn chronised with the volume acquisition (Fig. 1).

\section{Insert Figure 1 about he re}


Image pre-processing

fMRI data were analysed using statistical parametric mapping software (SPM2, Wellcome Department of Cognitive Neurology, London, http://www.fil.ion.ucl.ac.uk/spm). All functional volumes were time corrected, motion corrected by spatial realignment to the first volume, and normalised to the MNI reference brain (courtesy of the Montreal Neurological Institute). The normalised functional images were then spatially smoothed with an $8 \mathrm{~mm}$ full-width halfmaximum Gaussian kernel to accommodate intersubject anatomical variability.

\section{Statistical analysis}

The statistical analysis of the variations of the BOLD signal was based on the application of the general linear model to time series of the task-related functional activations (Friston et al., 1995). Trials for all three events (Novel, Deviant and Standard) were modelled separately by using a canonical hæmodynamic response function (HRF) and its first-order temporal derivative (Friston et al., 1998). The three standard sounds following a 'resting' period as well as the standard sounds following a rare stimulation (deviant or novel) were modelled as separate events. Contrast images (Novel-Standard and Deviant-Standard) consisting of statistical parametric maps (SPMs) of $t$ statistics at each voxel were then produced for each individual. These $\operatorname{SPM}\{t\}$ were entered into a second level group analysis to create an $\operatorname{SPM}\{F\}$, permitting inferences about condition effects across subjects that generalise to the population (i.e. 'random effects analysis'). $\operatorname{SPM}\{F\}$ statistics including both the HRF and its temporal derivative (corrected for non-sphericity) were thus computed for each contrast to examine areas of activation for the group as a whole (Control + Autism), with a threshold of $P<0.01$ false discovery rate (FDR) corrected for multiple comparisons (Genovese et al., 2002). Differences between groups were evaluated in a similar manner by computing for each 
contrast the $\operatorname{SPM}\{F\}$ statistics thresholded at $P<0.001$ uncorrected. Thus, the only difference between the two analyses (group as a whole and between groups comparison) was in the FDR correction which was applied for the population effects only. For each analysis the $F$ statistic was subsequently converted to a standard normal $z$ statistic for reporting. The locations of significant activations were expressed in Talairach coordinates (Talairach and Tournoux, 1988), using the nonlinear transformation procedure developed by M Brett (mni2tal: http://www.mrc-cbu.cam.ac.uk/Imaging/Common/mnispace.shtml).

\section{RESULTS}

\section{Deviance-detection}

Activation common to both groups

The contrast between Deviant and Standard sounds produced sign ificant activation of multiple brain regions in an analysis of data from both groups combined (Table 1). Generically activated regions included bilateral superior temporal gyri (STG, approximate Brodmann area [BA] 22) and bilateral middle temporal gyri (BA 21), the extent of activation being greater in the right hemisphere (Figure 2A). Activation was also observed in the right and left inferior frontal gyri (IFG, BA 44/45), the right inferior parietal lobules (IPL, BA 40), the right and left posterior cerebellum, the right medial dorsal thalamus and right head of the caudate, right cingulate gyrus and right medial temporal region.. In all these regions, the response to Deviant stimuli was greater than the response to Standard stimuli.

\section{Insert Table 1 and Figure 2 about here}


Activation differences between groups

The main group difference in response to deviant stimuli was found in the left anterior cingulate gyrus, which was more strongly activated by the control group than by children with autism (Table 2, Figure 4A). To a lesser extent, the left medial orbitofrontal region and the left inferior frontal gyrus also demonstrated greater magnitude of activation in controls than in children with autism (Figure 3A). No activation difference was found in the opposite direction (i.e. greater activation in the autism group as compared to the controls). Further analysis was performed within each group by computing $\operatorname{SPM}\{F\}$ statistics for the Deviant minus Standard contrast, in order to assess the activations in response to standard sounds and in response to deviant sounds in the anterior cingulate cortex. This showed that whereas Deviant sounds enhanced activation of this region in controls $(z=3.25 ; P<0.01$, uncorrected), children with autism showed the reverse pattern, with Deviant sounds showing less activation compared to the Standards ( $\mathrm{z}=3.15 ; P<0.01$, uncorrected). Notably, there was no evidence for any significant between-group difference in activation of auditory cortical regions.

\section{Insert Table 2 and Figure 3 about here}

\section{Novelty-detection}

Activation common to both groups

The most salient focus of activation by Novel sounds compared to Standard sounds was in the bilateral STG (Table 1, Figure 2B). This activation was more extensive in the right temporal cortex with additional significant signal increase in the right middle temporal gyrus (BA 21) and superior temporal pole (BA 38). Additional areas of activation were located in bilateral 
IFG (BA 44/45), bilateral temporo-parietal junction (BA 40/22), cingulate gyrus and right head of the caudate nucleus. In all these regions, the response to Novel stimuli was greater than the response to Standard stimuli.

Activation differences between groups

The main group differences in response to Novel stimuli were found in the left and right temporo-parietal junction (IPL and posterior STG), the right IFG and middle frontal gyrus, the left anterior cingulate gyrus, and the right anterior cerebellum (Table 2, Figure 3B). In all these regions, differential response to Novel stimuli was significantly attenuated in children with autism compared to controls. Further analysis in the anterior cingulate cortex did not show the reversed group patterns previously observed for deviance detection. It was again notable that there was no evidence for significant between-group difference in activation of auditory cortical regions.

\section{DISCUSSION}

This study is the first attempt to address the brain processes involved in auditory devianceand novelty-detection in typical children and in children with autism, using fMRI. As predicted, the results showed that despite normal processing of infrequent acoustic changes at the level of the auditory cortex, regions involved in involuntary attention switching during change detection displayed atypical activation in autism. Specifically lower activity was found in autism in the left anterior cingulate and the left inferior frontal region during deviance-detection, and in the left anterior cingulate, the bilateral temporo-parietal junction and the right frontal cortex during novelty-detection. Our results in typical children are 
consistent with those obtained in previous studies of auditory chan ge detection in adults. The find ings in the autism group are congruent with our hypothesis of atypical change detection.

\section{Deviance- detection}

Combining data from all volunteers, significant activation was seen in both the superior temporal gyrus and the inferior frontal gyrus during deviance-detection, confirming previous find ings from PET and fMRI studies in adu lts (Müller et al., 2002; Opitz et al., 2002; Doeller et al., 2003; Rinne et al., 2005). The current study confirmed that additional brain regions are involved in deviance-detection. Activation of the inferior parietal lobule and the temporoparietal junction has been previously identified in an adult fMRI study (Celsis et al., 1999). Our findings of involvement of the right head of the caudate nucleus, the cingulate gyrus and the cerebellum are consistent with PET data (Dittmann-Balcar et al., 2001), suggesting automatic subcortical processing of deviant auditory stimuli, and may reflect cerebellar involvement in the pre-attentional processes of prediction and preparation (Allen et al., 1997).

The larger activation in the right more than in the left temporal region during deviance detection accords with previous electrophysiological (Giard et al., 1995) and magnetoencephalographic (MEG) findings (Levänen et al., 1996). These MEG results suggest that two dipoles are necessary to model MMN activity in the right hemisphere. The additional right generator is located in the inferior parietal lobule/temporo-parietal junction and would be involved in a more global and non-specific change detection process (Levänen et al., 1996). This finding of more general processing in the temporo-parietal junction has been

confirmed in an fMRI study showing that the right posterior superior temporal gyrus is activated by brief periods of silence used as deviant events, and thus plays a specific role in 
the integration of sequential auditory events rather than in the analysis of stimulus features (Mustovic et al., 2003).

Comparison of our data with results from other studies remains difficult since there have not yet been other fMRI studies of the oddball paradigm in children. Thus we cannot establish clearly whether the highly significant activations found for the deviance contrast are related to the original paradigm we used (stimulus delivery in the 'silent' gap) or to the age of the population studied. rCBF has been shown to be elevated in children relative to adults in several developmental studies and is supposed to reach adult levels only between 15 and 19 years (Chiron et al., 1992; Takahashi et al., 1999). Slight variations in the BOLD response might thus be more clearly identified in younger populations. Moreover, deviance-detection elicits larger brain electrical responses in children than in adults (Csépé, 1995; Gomot et al., 2000), and this age dependence is likely to be associated with larger BOLD responses in the corresponding cerebral regions (Logothetis et al., 2001). One possible extension to our study would be to scan a group of typical adults using our paradigm in order to address this agerelated is sue.

The direct group comparison during deviance-detection showed a lower activation in the left anterior cingulate region in the autism group. This is an interesting finding as this area is thought to play a crucial role in stimulus evaluation (Bush et al., 2000). Anterior cingulate cortex is theorised to belong to a recency system that abandons older, stored information in order to capture new potentially relevant information (Ebmeier et al., 1995). The present results suggest that the hypo-activation in the left anterior cingulate cortex in children with autism compared to controls could actually reflect an inhibition of this region during deviance-detection. This interpretation is consistent with a recent, analogous result in the case of visual stimuli: children with autism deactivated the anterior cingulate gyrus when 
processing visual distractors incongruent to a target stimulus as contrasted with congruent distractors (Belmonte and Baron-Cohen, 2004). Furthermore, inhibition of activity in this area could explain the lower activation that we found in the left prefrontal cortex in autism, given that these two regions have extensive connections. As the anterior cingulate is involved in detection of non-routine situations and is thought to trigger the lateral prefrontal cortex to engage further attentional top-down cognitive processes (Carter et al., 2000), atypical inhibitory mechanisms in this region could prevent appropriate allocation of pre-attentional processes to changing events.

Interestingly, the anterior cingulate region has been highlighted in autism in independent studies using entirely unrelated measurement techniques. Convergent findings from SPECT (Ohnishi et al., 2000) PET (Haznedar et al., 1997, 2000), ${ }^{1}$ H-MRSI (Levitt et al., 2003), structural MRI (Abell et al., 1999; Haznedar et al., 2000) and post-mortem (Bauman and Kemper, 1994) studies support the idea that the cingulate gyrus displays both functional (hypoperfusion, hypometabolism) and anatomic (size reduction and increased cell density) abnormalities in autism. Moreover, diffusion tensor imaging (DTI) has recently demonstrated a reduction of fractional anisotropy in the white matter adjacent to the anterior cingulate gyri, suggesting a disruption of neural connections between this region and other brain structures (Barnea-Goraly et al., 2004).

Additionally, the current data indicate lower activation in the left inferior frontal gyrus in the autism group during deviance-detection. The frontal activity associated with the triggering of an attention switch during change detection is classically localised on the right hemisphere. However, evidence of the involvement of the left inferior frontal gyrus in the mismatch process has been provided by lesions studies (Alho et al., 1994; Alain et al., 1998) and fMRI studies (Doeller et al.,2003; Rinne et al., 2005), and in the present study we also found 
activation in the left frontal region in the pattern common to both groups. This leads us to suggest that the left inferior frontal gyrus could also house an attention switch mechanism associated with deviance-detection. Our results suggest that such mechanism that would enhance the automatic perception of potentially relevant deviant events is atypical in autism.

\section{Novelty-detection}

Novelty detection processes are elicited by salient, unexpected events, which are new each time. Our study reveals that the presentation of novel stimuli outside the focus of attention elicits significant functional activity in a network very similar to that involved in processing of deviant stimuli, both in controls and in children with autism. This network includes the superior temporal gyrus, the temporo-parietal junction, the superior and inferior frontal gyrus, the cingulate gyrus and the right medial dorsal thalamus and head of the caudate nucleus. However the activations were slightly more widespread in response to novel stimuli compared to deviant stimuli in the right inferior frontal region, and additional activations were seen in the left superior frontal gyrus, these frontal activations perhaps being associated with involuntary switching of attention toward the novel stimulus.

In the current study, novelty-detection (like deviance-detection) was characterised by more extensive activation in the right hemisphere (with two separate anatomical foci and larger cluster size), confirming the findings of the PET study reported by Müller et al. (2002). This right dominance could be due to the frequency complexity of the sounds we used for both deviant and novel stimuli (no simple sinuso idal sounds), which is more likely to be processed within the right superior temporal cortex (Wessinger et al., 2001). However this result is also consistent with the idea of right-hemisphere specialisation for the orienting of attention (Mesulam, 1998). 
In individuals with autism, along with atypical activity in the left anterior cingulate gyrus, novelty-detection elicited reduced activation in the left and right temporo-parietal region, and in the right inferior and middle frontal areas, compared to controls. These regions are described as belonging to the same novelty-detection circuit and are assumed to have a major role in attention-switching and in the distribution of attentional resources (Daffner et al., 2003). The temporo-parietal junction and the middle frontal region belong to the network of cerebral regions in which disruption of related white matter tracts has been demonstrated in autism using DTI (Barnea-Goraly et al., 2004). Moreover, measurement of cerebral glucose metabolism during rest demonstrates impairment in functional as sociation between these two regions in autism (Horwitz et al., 1988).

In the current study, atypical activations within the novelty-detection network in children with autism were particularly noteworthy in the temporo-parietal junction. This region is assumed to be involved in the pre attentive gating mechanism that determines the extent to which unattended novel sounds enter awareness (Jääskeläinen et al., 2004). However, it has been shown that top-down attentional processes influence such pre attentive mechanisms. While habituation to repeated novel stimuli occurs in the frontal regions regardless of the focus of attention, it occurs in the parietal region when attention is directed away from the oddball stimuli (Cycowicz and Friedman, 1998). Thus, although all the volunteers in our experiment received the same instruction to focus on the video, one cannot exclude the possibility that people with autism were more absorbed in the movie they were watching and thus engaged fewer attentional resources in the processing of the auditory stimuli than the controls. Such a unimodal style of attention could have resulted in the lower responsiveness of the temporoparietal region to novel auditory events in autism. The atypical activation in the temporoparietal junction in autism suggests unusual reactivity of brain regions usually involved in 
change detection, as if the autistic brain became 'hermetic' (or impenetrable) to novelty in one sensory modality while involved in a processing in another modality. Such a view if correct would be concordant with attention-switching difficulties reported in clinical observations. Controlling for such attentional effects may be possible if a concurrent visual task was presented to the participants while the sound sequence is delivered.

The analysis of new events occurring outside the focus of attention is fundamental to adaptive functioning. Sensory events that are important for survival demand further analysis according to their relevance. The current results suggest people with autism may have some difficulty in involuntary attention switching when processing unexpected infrequent stimuli. Mesulam's (1998) view of 'novelty seeking' provides an interesting framework for discussing these findings. According to this view, the bias for perceiving sameness and emitting automatic responses would represent 'a default mode' of brain function, and would lead to behaviours that can be designated instinctual, stereotyped or stimulus-bound. This mode would enhance efficiency and reliability but also increase premature closure, perseveration and rigidity. Thus one evolutionary improvement has been to develop specialised neural circuits dedicated to the rapid detection of unfamiliar events (Mesulam, 1998). The results discussed here suggest that these networks are atypical in autism. This could lead to a 'default mode' of brain functioning producing conservative and stereotyped behaviours. In this case, 'avoidance' of change would represent an adaptive behaviour by a brain aiming to keep the environment as predictable as possible, and might be considered as a 'secondary dysfunction' of autism (Belmonte et al., 2004).

\section{Conclusion}


To summarise, our study found atypical activation of brain regions involved in involuntary attention switching associated with automatic detection of changing auditory events in children with autism. We demonstrated abnormalities in the cingulate gyrus regardless of the type of infrequent stimuli, and an additional reduction of activation in temporo-parietal and frontal regions dedicated to novelty-detection. This difficulty in modulating the processing of incoming new events may contribute to the clinical reports in autism of a tendency to ignore novel stimuli when they occur outside the focus of attention. This atypical response to changing events could play a role in the difficulty presented in people with autism in modifying expectancies in response to rare sensory information, and may throw light on the neurophysio logical process underlying 'resistance to change' in autism.

\section{Acknowledgements}

M. G. was supported by grants from the Fondation pour la Recherche Medicale, the 'Region Centre' and the 'Fondation d'Entreprise France Telecom'. F.A.B. was supported by a Merck Sharp and Dohme Post-doctoral Fellowship award. M.K.B. was supported by Cure Autism Now and C.A. by the National Alliance for Autism Research. S.B-C and E.B. were supported by the UK Medical Research Council. The Wolfson Brain Imaging Centre is supported by an MRC Co-operative Group grant. We thank José Alcantara, Maarten VanCasteren, Richard Henson, Christian Schwartzbauer and Bernhard Müller for their contribution to the experimental paradigm, Ruth Bisbrown-Chippendale for technical assistance with MRI data acquisition, Howard Ring for valuable discussion of the study, and most of all the participants and their parents for their time and effort in this study. 


\section{References}

Abell, F., Krams, M., Ashburner, J., Passingham, R., Friston, K., Frackowiak, R., et al., 1999. The neuroanatomy of autism: a voxel-based whole brain analysis of structural scans. Neuroreport 10(8), 1647-1651.

Alain, C., Woods, D.L., Knight, R.T., 1998. A distributed cortical network for auditory sensory memory in humans. Brain Res. 812(1-2), 23-37.

Alho, K., 1995. Cerebral generators of mismatch negativity (MMN) and its magnetic counterpart (MMNm) elicited by sound changes. Ear Hear. 16, 38-51.

Alho, K., Woods, D.L., Algazi, A., Knight, R.T., Näätänen, R., 1994. Lesions of frontal cortex diminish the auditory mismatch negativity. Electroencephalogr. Clin. Neurophysiol. 91, $353-362$.

Alho, K., Winkler, I., Escera, C., Huotilainen, M., Virtanen, J., Jääskeläinen, I.P., et al., 1998. Processing of novel sounds and frequency changes in the human auditory cortex: magnetoencephalographic recordings. Psychophysiology 35(2), 211-224.

Allen, G., Buxton, R.B., Wong, E.C., Courchesne, E., 1997. Attentional activation of the cerebellum independent of motor involvement. Science 275, 1940-1943.

American Psychiatric Association (APA), 2000. Diagnostic and Statistical Manual-IV-Text revision. American Psychiatric Association, Washington, DC.

Barnea-Goraly, N., Kwon, H., Menon, V., Eliez, S., Lotspeich, L., Reiss, A.L., 2004. White matter structure in autism: preliminary evidence from diffusion tensor imaging. Biol. Psychiatry 55(3), 323-326.

Bauman, M.L., Kemper, T.L. (Eds.), 1994. Neuroanatomic observations of the brain in autism. In: The neurobiology of autism. Johns Hopkins University Press, Baltimore, pp.119-145.

Belmonte, M.K., Cook, E.H., Anderson, G.M., Rubenstein, J.L., Greenough, W.T., BeckelMitchener, A., et al., 2004. Autism as a disorder of neural information processing: 
directions for research and targets for therapy. Mol. Psychiatry 9(7), 646-663. Unabridged edition at http :/www.cureautismnow .org/media/3915.pdf.

Belmonte, M.K., Baron-Cohen, S., 2004. Normal sibs of children with autism share negative frontal but not positive sensory abnormalities: preliminary evidence from fMRI during processing of visual distractors. Program $\mathrm{N}^{\circ} 582.10$. 2004 Abstract Viewer/Itinerary Planner. Society for Neuroscience, Washington, DC, Online.

Bomba, M.D., Pang, E.W., 2004. Cortical auditory evoked potentials in autism: a review. Int. J. Psychophysiol. 53(3), 161-169.

Bush, G., Luu, P., Posner, M.I., 2000. Cognitive and emotional influences in anterior cingulate cortex. Trends Cogn. Sci. 4(6), 215-222.

Carter, C.S., MacDonald, A.M., Botvinick, M., Ross, L.L., Stenger, V.A., Noll, D., et al., 2000. Parsing executive processes: strategic vs. evaluative functions of the anterior cingulate cortex. Proc. Natl. Acad. Sci. USA 97(4), 1944-1948.

Celsis, P., Boulanouar, K., Doyon, B., Ranjeva, J., Berry, I. Nespoulous, J., et al., 1999. Differential fMRI responses in the left posterior superior temporal gyrus and left supramarginal gyrus to habituation and change detection in syllables and tones. NeuroImage 9, 135-144.

Ceponiene, R., Lepisto, T., Shestakova, A., Vanhala, R., Alku, P., Näätänen, R., et al., 2003. Speech-sound-selective auditory impairment in children with autism: they can perceive but do not attend. Proc. Natl. Acad. Sci. USA 100(9), 5567-5572.

Cheour-Luhtanen, M., Alho, K., Sain io, K., Rinne, T., Reinikainen, K., Pohjavuori, M., et al., 1996. The ontogenetically earliest discriminative response of the human brain. Psychophysiology 33, 478-481.

Chiron, C., Raynaud, C., Maziere, B., Zilbovicius, M., Laflamme, L., Masure, M.C., et al., 1992. Changes in regional cerebral blood flow during brain maturation in children and adolescents. J. Nucl. Med. 33(5), 696-703. 
Courchesne, E., Kilman, B.A., Galambos, R., Lincoln, A.J., 1984. Autism: processing of novel auditory information assessed by event-related brain potentials. Electroencephalogr. Clin. Neurophysiol. 59(3), 238-248.

Courchesne, E., Lincoln, A., Kilman, B.A., Galambos, R., 1985. Event-related brain potential correlates of the processing of novel visual and auditory information in autism. J. Autism Dev. Disord. 15, 55-76.

Csepe, V., 1995. On the origin and development of the mismatch negativity. Ear Hear. 16(1), 91-104.

Cycowicz, Y.M., Friedman, D., 1998. Effect of sound familiarity on the event-related potentials elicited by novel environmental sounds. Brain Cogn. 36(1), 30-51.

Daffner, K.R., Scinto, L.F., Weitzman, A.M., Faust, R., Rentz, D.M., Budson, A.E., et al., 2003. Frontal and parietal components of a cerebral network mediating voluntary attention to novel events. J. Cogn. Neurosci. 15(2), 294-313.

Dittmann-Balcar, A., Juptner, M., Jentzen, W., Schall, U., 2001. Dorsolateral prefrontal cortex activation during automatic auditory duration-mismatch processing in humans: a positron emission tomography study. Neurosci. Lett. 308(2), 119-122.

Doeller, C., Opitz, B., Mecklinger, A., Krick, C., Reith, W., Schröger, E., 2003. Prefrontal cortex involvement in preattentive auditory deviance detection: neuroimaging and electrophysiological evidence. NeuroImage 20, 1270-1282.

Downar, J., Crawley, A.P., Mikulis, D.J., Davis, K.D., 2002. A cortical network sensitive to stimulus salience in a neutral behavioral context across multiple sensory modalities. J. Neurophysiology 87, 615-620.

Downar, J., Crawley, A.P., Mikulis, D.J., Davis, K.D., 2000. A multimodal cortical network for the detection of changes in the sensory environment. Nat. Neurosci. 3(3), 277-283.

Ebmeier, K., Steele, J., MacKenzie, D., O’Carroll, R., Kydd, R., Glabus, M., et al., 1995. Cognitive brain potentials and regional cerebral blood flow equivalents during two- and 
three-sounds auditory 'oddball tasks'. Electro encephalogr. Clin. Neurophysiol. 95, 434443.

Escera, C., Alho, K., Winkler, I., Näätänen, R., 1998. Neural mechanisms of involuntary attention switching to novelty and change in the acoustic environment. J. Cogn. Neurosci. $10(5), 590-604$.

Escera C., Alho K., Schroger E., Winkler I., 2000. Involuntary attention and distractibility as evaluated with event-related brain potentials. Audiol. Neurootol. 5, 151-66.

Ferri, R., Elia, M., Agarwal, N., Lanuzza, B., Musumeci, S.A., Pennisi, G., 2003. The mismatch negativity and the P3a components of the auditory event-related potentials in autistic low-functioning subjects. Clin. Neurophysiol. 114(9), 1671-1680.

Friston, K.J., Holmes, A.P., Worsley, K.J., Poline, J.B., Frith, C.D., Frackowiak, R.S., 1995. Statistical parametric maps in functional imaging: a general linear approach. Hum. Brain Mapp. 2, 189-210.

Friston, K.J., Fletcher, P., Josephs, O., Holmes, A.P., Rugg, M.D., Turner, R., 1998. Eventrelated fMRI: characterizing differential responses. NeuroImage 7, 30-40.

Frith, U., 1970. Studies in pattern detection in normal and autistic children. II. Reproduction and production of color sequences. J. Exp. Child Psychol. 10(1), 120-135.

Genovese, C.R., Lazar, N.A., Nichols, T., 2002. Thresholding of statistical maps in functional neuroimaging using the false discovery rate. NeuroImage 15(4), 870-878.

Giard, M.H., Perrin, F., Pernier, J., Bouchet, P., 1990. Brain generators implicated in the processing of auditory stimulus deviance: A topographic event-related potential study. Psychophysiology 27, 627-640.

Giard, M.H., Lavikainen, J., Reinikainen, K., Perrin, F., Bertrand, O., Thévenet, M., Pernier, J., Näätänen, R., 1995. Separate representation of stimulus frequency, intensity, and duration in auditory sensory memory. J of Cogn. Neurosci. 7, 133-143. 
Gomot, M., Giard, M.H., Roux, S., Barthelemy, C., Bruneau, N., 2000. Maturation of frontal and temporal components of mismatch negativity (MMN) in children. Neuroreport 11(14), 3109-3112.

Gomot, M., Giard, M.H., Adrien, J.L., Barthelemy, C., Bruneau, N., 2002. Hypersensitivity to acoustic change in children with autism: Electrophysiological evidence of left frontal cortex dysfunctioning. Psychophysio logy 39, 577-584.

Haznedar, M.M., Buchsbaum, M.S., Metzger, M., Solimando, A., Spiegel-Cohen, J., Hollander, E., 1997. Anterior cingulate gyrus volume and glucose metabolism in autistic disorder. Am. J. Psychiatry 154(8), 1047-1050.

Haznedar, M.M., Buchsbaum, M.S., Wei, T.C., Hof, P.R., Cartwright, C., Bienstock, C.A., et al., 2000. Limbic circuitry in patients with autism spectrum disorders studied with positron emission tomography and magnetic resonance imaging. Am. J. Psychiatry 157(12), 1994-2001.

Horwitz, B., Rumsey, J.M., Grady, C.L., Rapoport, S.I., 1988. The cerebral metabolic landscape in autism. Intercorrelations of regional glucose utilization. Arch. Neurol. 45(7), $749-755$.

Jääskeläinen, I.P., Ahveninen, J., Bonmassar, G., Dale, A.M., Ilmoniemi, R.J., Levän en, S., et al., 2004. Human posterior auditory cortex gates novel sounds to consciousness. Proc. Natl. Acad. Sci. USA 101(17), 6809-6814.

Kanner, L., 1943. Autistic disturbances of affective contact. Nervous Child 2, 217-250.

Kemner, C., Verbaten, M.N., Cuperus, J.M., Camfferman, G., Van Engeland, H., 1995. Auditory event-related brain potentials in autistic children and three different control groups. Biol. Psychiatry 38, 150-165.

Knight, R., 1996. Contribution of human hippocampal region to novelty detection. Nature $383,256-259$. 
Kobayashi, R., Murata, T., 1998. Behavioral characteristics of 187 young adults with autism. Psychiatry Clin. Neurosci. 52, 383-390.

Levänen, S., Ahonen, A., Hari, R., McEvoy, L., Sams, M., 1996. Deviant auditory stimuli activate human left and right auditory cortex differently. Cereb. Cortex 6(2), 288-296.

Levitt, J.G., O'Neill, J., Blanton, R.E., Smalley, S., Fadale, D., McCracken, J.T., et al., 2003. Proton magnetic resonance spectroscopic imaging of the brain in childhood autism. Biol. Psychiatry 54(12), 1355-1366.

Liebenthal, E., Ellingson, M., Spanaki, M., Prieto, T., Ropella, K., Binder, J., 2003. Simultaneous ERP and fMRI of the auditory cortex in a passive oddball paradigm. Neuroimage 19, 1395-1404.

Lincoln, A., Courchesne, E., Harms, L., Allen, M., 1993. Contextual probability evaluation in autistic, receptive developmental language disorder and control children: ERP evidence. J. Autism Dev. Disord. 23, 37-58.

Logothetis, N.K., Pauls, J., Augath, M., Trinath, T., Oeltermann, A., 2001. Neurophysiological investigation of the bas is of the fMRI signal. Nature 412, 150-157.

Lord, C., Rutter, M., LeCouteur, A., 1994. Autism Diagnostic Interview Revised: A revised version of a diagnostic interview for caregivers of individuals with possible pervasive developmental disorders. J. Autism Dev. Disord. 24, 659-685.

Malvy, J., Barthelemy, C., Damie, D., Lenoir, P., Bodier, C., Roux, S., 2004. Behaviour profiles in a population of infants later diagnosed as having autistic disorder. Eur. Child Adolesc. Psychiatry 13(2), 115-122.

Mathiak, K., Rapp, A., Kircher, T., Grodd, W., Hertrich, I., Weiskopf, N., et al., 2002. Mismatch responses to randomized gradient switching noise as reflected by fMRI and whole-head magnetoencephalography. Hum. Brain Mapp. 16, 190-195.

Mesulam, M.M., 1998. From sensation to cognition. Brain 121, 1013-1052. 
Molholm, S., Martinez, A., Ritter, W., Javitt, D.C., Foxe, J.J., 2005. The neural circuitry of pre-attentive auditory change-detection: an fMRI study of pitch and duration mismatch negativity generators. Cereb. Cortex 15(5), 545-51.

Müller, B., Jüptner, M., Jentzen, W., Müller, S., 2002. Cortical activation to auditory mismatch elicited by frequency deviant and complex novels sounds: A PET study. NeuroImage 17, 231-239.

Mustovic, H., Scheffler, K., Di Salle, F., Esposito, F., Neuhoff, J.G., Hennig, J., et al., 2003. Temporal integration of sequential auditory events: silent period in sound pattern activates human planum temporale. NeuroImage 20(1), 429-434.

Näätänen, R., 1992. Attention and Brain Function. Erlbaum, Hillsdale, NJ.

Näätänen R., Jacobsen T., Winkler I. 2005. Memory-based or afferent processes in mismatch negativity (MMN): A review of the evidence. Psychophysiology 42(1),25-32.

Novitski, N., Alho, K., Korzyukov, O., Carlson, S., Matinkauppi, S., Escera, C., et al., 2001. Effects of acoustic gradient noise from functional magnetic resonance imaging on auditory processing as reflected by event-related brain potentials. NeuroImage 14, 244251.

Ohnishi, T., Matsuda, H., Hashimoto, T., Kunihiro, T., Nishikawa, M., Uema, T., et al., 2000. Abnormal regional cerebral blood flow in childhood autism. Brain 123, 1838-1844.

Opitz, B., Mecklinger, A., Friederici, A., Von Cramon, D.Y., 1999a. The functional neuroanatomy of novelty processing: integrating ERP and fMRI results. Cereb. Cortex 9, 379-391.

Opitz, B., Mecklinger, A., Von Cramon, D.Y., Kruggel, F., 1999b. Combining electrophysiological and hemodynamic measures of the auditory oddball. Psychophysiology 36, 142-147. 
Opitz, B., Rinne, T., Mecklinger, A., Von Cramon, D.Y., Schröger, E., 2002. Differential contribution of frontal and temporal cortices to auditory change detection: fMRI and ERP results. NeuroImage 15, 167-174.

Picton T.W., Alain C., Otten L., Ritter W., Ach im A. 2000. Mismatch negativity: different water in the same river. Audiol. Neurootol. 5, 111-39.

Rinne, T., Degerman, A., Alho, K., 2005. Superior and inferior frontal cortices are activated by infrequent sound duration decrements: An fMRI study. NeuroImage, Advance on line publication.

Rogers, S.J., Hepburn, S., Wehner, E., 2003. Parent reports of sen sory symptoms in toddlers with autism and those with other developmental disorders. J. Autism Dev. Disord. 33(6), 631-642.

Sabri, M., Kareken, D., Dzemidzic, M., Lowe, M., Melera, R., 2004. Neural correlates of auditory sensory memory and automatic change detection. NeuroImage 21, 69-74.

Schall, U., Johnston, P., Todd, J., Ward, P.B., Michie, P.T., 2003. Functional neuroanatomy of auditory mismatch processing: an event-related fMRI study of duration-deviant oddballs. Neuroimage 20(2), 729-36.

Schröger, E., 1996. A neural mechanism for involontary attention shifts to changes in auditory stimulation. J. Cogn. Neurosci. 8, 527-539.

Seri, S, Cerquiglini, A., Pisani, F., Curatolo, P., 1999. Autism in tuberous sclerosis: evoked potential evidence for a deficit in auditory sensory processing. Clin. Neurophysiol. 110(10), 1825-1830.

Takahashi, T., Shirane, R., Sato, S., Yoshimoto, T., 1999. Developmental changes of cerebral blood flow and oxygen metabolism in children. Am. J. Neuroradiol. 20(5), 917-922.

Talairach, J., Tournoux, P., (Eds.) 1988. Co-planar strereotaxic atlas of the human brain. Thieme Verlag, Stuttgart. 
Tervaniemi, M., Schröger, E., Saher, M., Näätänen, R., 2000. Effects of spectral complexity and sound duration on automatic complex-sound pitch processing in humans - a mismatch negativity study. Neurosci. Lett. 290(1), 66-70.

Wechsler Abbreviated Scale of Intelligence Manual, 1999. The Psychological Corporation, San Antonio.

Wessinger, C.M., VanMeter, J., Tian, B., Van Lare, J., Pekar, J., Rauschecker, J.P., 2001. Hierarchical organization of the human auditory cortex revealed by functional magnetic reson ance imaging. J. Cogn. Neurosci. 13(1), 1-7.

Wing, L., 2003. The Autistic Spectrum: A Guide for Parents and Professionals, 2nd Ed. Constable and Robinson, London. 


\section{Tables:}

Table 1: Main regions demonstrating significant activation by Deviant sounds compared to Standard sounds, and Novel sounds compared to Standard sounds, in all subjects $(\mathrm{N}=24$; control + autism; $P<0.01$, FDR corrected).

Table 2: Brain locations of significant difference between groups in response to Deviant sounds and $\operatorname{Novel}(\mathrm{N}=12$ in each group; $P<0.001$, uncorrected).

\section{Figures:}

Figure 1: Schematic illustration of the synchronization between the sequence of acquisition and the sequence of stimulation. A pulse was sent by the scanner at every slice and a stimulus was delivered every 5 pulses, after a delay of $50 \mathrm{~ms}$. Auditory stimuli were thus delivered exactly in the 'silent' gap between the acquisitions of two slices.

Fig 2: Group activation map showing brain regions activated on average over all subjects (N $=24$; Control + Autism) by processing of (A) Deviant sounds compared to Standard sounds; and (B) Novel sounds compared to Standard sounds. Voxels with activation significant at $P<0.01(\mathrm{~F}=9.14)$, FDR corrected for multiple comparison $\mathrm{s}$ are shown; $\mathrm{R}=$ right hemisphere; $\mathrm{L}=$ left hemisphere.

Fig 3: Group difference map showing brain regions differentially activated between groups (Control > Autism; $\mathrm{N}=12$ in each group) by processing of (A) Deviant sounds compared to Standard sounds; and (B) Novel sounds compared to Standard sounds. Voxels with activation significant at $P<0.001(\mathrm{~F}=8.12)$ are shown; $\mathrm{R}=$ right hemisphere; $\mathrm{L}=$ left hemisphere. 
Fig 4: Plots show adjusted fMRI activity (arbitrary units) in the left anterior cingulate for each child with autism (red rhombus) and for each healthy control (blue dot) in (A) the Deviant versus Standard contrast ( $x=-13, y=19, z=23)$ and in $(B)$ the Novel versus Standard contrast $(x=-15, y=17, z=23)$. 


\section{${ }^{*}$ Response to Reviews}

\section{Response to reviewers}

\section{Reviewer 2:}

The manuscript starts by introducing MMN and P3a which have been associated in previous literature with well defined brain processes: it is assumed that the supratemporal MMN reflects auditory change detection, frontal component of MMN is typically connected to initiation of switching attention (this is the prevailing theory) and P3a is associated with attention switch itself. The authors fail to define and use these key concepts in a systematic way.

We agree that we have to be as clear and as precise as possible when defining these concepts. We think the only issue here regards terminology. Both frontal and temporal components of the MMN are evoked during the processing of change. The whole mechanism thus includes both detection of a mismatch at the auditory level, and involuntary orientation of attention toward this change at the frontal level, the chronology of these activations still being under discussion.

Referee 2 suggested that the supratemporal MMN reflects pre-attentional auditory change detection and that the frontal component of MMN is related to initiation of the attention switch. Basically we agree, but in order to be fully accurate one should add that 'frontal component of MMN is related to initiation of the attention switch associated with change detection.' Moreover it seems that referee 2 consider that this frontal activity during deviance detection is not 'pre attentional'. This is confusing as this component has been recorded even in comatose patients.

Anyway, we improved the manuscript according to this criticism.

The authors are mainly interested in the comparison of two groups of children. However, the manuscript is, at least to some degree, based on the idea that the brain processes in children are similar to those in adults. The authors conclude that STG and IFG activations in children are in line with adult data but that additional areas are involved in auditory change detection and control of attention in children. Please elaborate on this.

What are the additional areas (the discussion says that caudate and cerebellum are included in these but you showed additional activation in many other areas, be systematic)? Is cingulate activation reported previously or is this a new finding?

This has been clarified (see $\mathrm{p} 14$ paragraph2). The activations we report in children have been reported in adults also in previous studies.

Discussion goes too far away from the data (are the group differences real or caused by differences in the task?). The authors do not seem to realize the value of their study as "first attempt". The Discussion should be more concise.

Specific comments:

Abstract

1) "passive detection" is really a confusing term. Explain what was done (sounds were presented while the subjects were...). Report activation to the deviants and novels: E.g, In both groups, unattended deviants...

'in a no-task condition ' now replaces 'passive'.

2) "During novelty-detection children", comma have been added

3) The sentence 'This study confirms previous evidence from ERP....' has been rephrased in two separate sentences: 'This study confirms previous evidence from ERP studies of atypical brain function related to automatic change detection in autism. Abnormalities involved a cortical network known to have a role in attention-switching and attentional resource distribution.'

Introduction 
4) The term 'automatic' is ill-defined and might have different connotations depending on the background of the reader. Please, do not use this term in this context (MMN, auditory change detection). Instead, explain that you are studying processing of acoustic stimuli when attention is directed away from the sounds. After this is explained, 'preattentive' might be used if needed.

\section{'Automatically' has been removed.}

5) The first paragraph of Introduction is really confusing. 'Automatic processing' (i.e., preattentive processing) and auditory change detection are well defined concepts that have nothing to do with social situations or communication. The first paragraph should describe autism and behavior typical to autism without referring to these concepts.

Please note that at this very first step of the manuscript we are not referring to any MMN or auditory change detection processes. We are introducing the fact that wherever you are, whatever you do, there is an adaptive functioning that allows you to detect changing events. This ability is particularly important when you are engage in a rapidly changing social situation. This involves behaviors that could be observed ecologically, that did exist before Näätänen 'discovered' the MMN and that are impaired in people with autism.

6) Please define or remove "pattern imposition"

This is now better explained: 'Finally, when left to their own devices, children with classic autism tend to compulsively create patterns, which may reflect a strong desire to impose control over changing events.'

7) In the second paragraph you fail to distinguish between auditory change detection and involuntary control of attention. This confusion is evident throughout the manuscript. It seems (although I am not sure about this) that you assume that auditory change detection in auditory cortex should not be affected by autism (e.g., in Discussion you write "As predicted, the results showed that despite normal processing of infrequent acoustic changes at the level of the auditory cortex,..") but that involuntary control of attention might be affected. I am sure that you are familiar with the assumed functional roles of supratemporal MMN, frontal MMN, and P3a and how these relate to auditory change detection and involuntary control of attention. Write accordingly.

Please, note that there was no idea of 'preattentive auditory change detection (MMN)' in this paragraph which aimed to introduce the fact that people with autism display major difficulties to deal with change (in general) and that they also display unusual behaviors in response to changing sensory events. We are describing clinics of autism and at this behavioral level it is not possible to distinguish between auditory change detection and involuntary switching of attention.

Only from the 3rd paragraph, we explain that one way to explore change processing at a very elementary ('perceptive') level is to record MMN, P3a using an oddball paradigm.

We are convinced that this progression, from clinics to cognitive processes is important when you aim to address a psychiatric disease.

8) By the third paragraph the reader is lost with the lack of systematic definition of the key concepts. What do you mean by "change detection"? "The neural correlates of change detection" which happens in social situations? You have to define the key concepts carefully. You have to explain that auditory change detection is assumed to be preattentive. You have to explain that recording of MMN/N1 enhancement does not require active participation from the subject and that it is generally assumed that attention does not change these responses (in typical experimental settings).

'pre-attentive ' has been added as well as 'It is assume to reflect a pre-attentional change detection process and its recording does not require active participation from the subject.'

9) The confusion goes on in the fourth paragraph: What is the subject doing during "detection of deviant stimuli"? Please do not assume that the reader will understand what you mean anyway. This is a rather complicated study (auditory change detection, involuntary control of attention, fMRI, children, autism). You have to explicitely describe the main ideas. 
We previously used the term 'passive detection' of deviant stimuli in order to simply describe the idea that attention was not focused on the stimuli. This expression was removed as suggested by referee 2 in the previous revision. We thus added 'in no-task condition'.

10) Your theoretical introduction to MMN and auditory change detection is poor.

-you want to say that (1) previous extensive ERP literature has identified an auditory cortex response (MMN) that is associated with preattentive detection of acoustic changes, i.e., auditory cortex MMN is associated with auditory change detection (please do not mess this up with loosely mentioning 'auditory sensory memory'), (2) it has been suggested that MMN might have an additional generator in the frontal lobes (please cite more experimental studies in addition to Giard 90) and that this has been associated with initiation/triggering of involuntary switching of attention to the detected changes (please do not mess this up by writing that this frontal component is associated with 'pre-attentional processing of deviance')

This paragraph has been rewritten: 'The ERP response evoked by any deviant stimuli is called Mismatch Negativity (MMN). It is assume to reflect a pre-attentional change detection process and its recording does not require active participation from the subject. MMN is thought to reflect the automatic detection of a difference between the active sensory memory trace of the recent repetitive event (the standard) and an incoming deviant stimulus (Näätänen, 1992; Näätänen et al., 2005). Several studies using different methods have identified cortical generators of MMN (Picton et al. 2000). The main MMN component is generated bilaterally in the supratemporal part of the auditory cortex and might be associated with acoustic change detection (see Alho, 1995 for review). An additional generator has been identified in the frontal region (Giard et al., 1990; Alho et al., 1994, Alain et al., 1998) which may be related to initiation of an involuntary attention switch toward the detected change (Giard et al., 1990; Näätänen, 1992; Schröger, 1996).

11) I am not sure what you mean by "supratemporal part of the auditory cortex". How about 'auditory areas in the supratemporal lobe' or 'supratemporal cortex?

The auditory areas spread over the supratemporal region and the lateral part of the temporal lobe. Here we refer to the part located in the supratemporal plane. (To our knowledge there is no such thing as 'supratemporal lobe' ).

12) Remove "scalp current density mapping" (several occurrences) from the Introduction (this is an fMRI study, this detail of ERP methodology is not relevant or interesting here).

The term SCD had been added in order to respond to the criticism made in the previous version by referee 2 (see comment and respond just below). Now this has to be removed. After this suppression, the sentence might be not accurate enough.

This is not detail of ERP methodology. SCD is one of the only ways to distinguish between temporal and frontal components of the MMN, which is relevant here as fMRI will allow to better localizing the brain regions involved in the generation of these components.

13) "Mechanisms underlying MMN are apparent early in development and are assumed to be similar across the lifespan"

-here you want to say that MMM can be recorded in children and adults and thus the underlying brain mechanisms are assumed to be similar across lifespan (Mechanism underlying MMN are not 'apparent' in newborns, but MMN can recorded in newborns)

This has been corrected. 'MMN can be recorded early in development and mechanisms underlying this response are assumed to be similar across the lifespan'

14) "A few MMN studies have been conducted with people with autism, and these have yielded contradictory findings."

- nobody 'conducts MMN studies' on autism(I hope...)

Please, note that we never wrote 'on autism', but we did wrote 'with people with autism'. We replaced the beginning of the sentence by: 'A few studies on auditory change detection mechanism underlying MMN have been conducted in people with autism'. 
-you want to say that a few previous studies have examined whether autism affects the auditory change detection mechanism underlying MMN or something of that nature

We didn't exactly want to say something like that. Autism, at the moment, is only defined as a behavioral syndrome. We do not consider autism as 'an event' (like a fever for example) that could affect MMN mechanism. Rather we do consider that the brain of people with autism developed in a particular way that lead to distortion in its structure and its functioning. Studying electrophysiological indices (reflecting specific brain processing) could provide information about these structural and functional abnormalities.

15) Introduction to P3a is confusing. It would be better to start the paragraph with "P3a response is thought to be associated with involuntary switching of attention..." and then to explain that P3a is elicited by novels but it can be elicited by small deviants too.

As P3a is not systematically recorded in response to small deviants, we think that perhaps this last bit is not necessary here and could be confusing for non expert readers.

You use 'detection' to denote preattentive processing but then you mess this up by using this term also in an active task ("novel-target detection"). Please define (explain what the subjects is doing etc) your concepts carefully (auditory change detection, oddball, 'automatic' etc) and use the terms systematically.

The introduced literature does not justify the conclusion that reduced P3a in autism is somehow related to "...difficulty in selecting relevant information" (whatever it means in this context).

'Active' has been added.

"...difficulty in selecting relevant information" has been removed.

16) "the deep and superficial anatomical structures" has been replaced by 'sub cortical and cortical structures'

17) "in typical populations/adults" -> normal populations/healthy adults (occurs in many parts of the ms)

This term was chosen on purpose. It is currently used in pathological studies when you want to distinguish between 'normal controls' and people with autism with 'atypical' brain development.

18) Spelling: Ceponiene

We added è instead of $\mathrm{e}$ at the end of the name, and 'Č' instead of ' $\mathrm{C}$ '.

19) "Automatic auditory change-detection has recently been studied in typical adults using fMRI"

-Cite the papers (there are more than 10 relevant fMRI studies). At least cite the ones published in 2004/5 (Molholm, Rinne etc):

Rinne and Molholm have been added at this level, but note that most of the papers are cited in the following sentences and that Molholm's paper was not published at the time when we submitted this manuscript.

20) "Despite the acoustic noise of fMRI,..."

-There are several ways to deal with the scanner noise. Scanner noise affects detection only if it is present during the stimulation. Please be more accurate.

'Despite the contamination of the stimuli by the acoustic noise...' has been added.

21) Moreover, although fMRI noise alters sensory response in auditory cortex [REF MISSING], it does not affect the change-detection response (Novitski et al., 2001).

The reference of Novitski et al., 2001 applies to the whole sentence.

-This is one example of the confusing way you use the key concepts: "change-detection response" is not defined; do you mean MMN, P3a or perhaps N1 enhancement? 
We didn't want to go back to electrophysiological responses at this step of the manuscript, as we are now talking about brain processes and fMRI studies. But as required we added: 'the change-detection response (MMN) (Novitski et al., 2001).'

22) Various fMRI block paradigms have been developed to study change detection, using the classical oddball paradigm

-paradigms using paradigms?

Replaced by 'block design fMRI studies".

I associate "classical oddball" with active detection of targets, please define.

As referee 2 has let us know that the term 'passive' was not adapted, we encountered some difficulties to define in a simple way this oddball paradigm involving 'no active detection of targets'. Would 'passive oddball' (a quite currently used expression which is straightforward and understood by most of the people) be all right? This term is the most commonly used and describes exactly our paradigm.

23) A few have highlighted the involvement of the other brain regions known to be associated with the mismatch process. - > ... involvement of the other brain regions in the processing of infreq sound changes.

-please cite the recently published studies too

This has been replaced

24) I would not make the (vague) point about ER vs. blocked designs. ER fMRI is no magic and you do not gain any extra 'points' with it. The issue of ER vs. blocked design in detecting IFC activation might be something to bring up in Discussion but not here.

In this context ER-fMRI is more suitable than block design, because event(-locked) responses can be modelled as short, rapid events, reflecting small but also more precise variations in the bold response. Thus, we firmly believe that this way of processing the data is particularly adapted to study 'early" information processing. Schall et al. 2003 have compared event-related and block analysis of their data and they showed that more brain regions were activated when using event-related analysis.' This reference has been added.

In this paragraph we justified the choice of the design we have used in this study. We think that it wouldn't have been logical to discuss that after the method section.

$25)$ "to test the hypothesis of atypical brain activation during automatic acoustic change detection in children with autism;"

-what hypothesis? Please be more specific.

Here we did not want to expose a more specific hypothesis (i.e. under/over activation) that's why we used the terms 'atypical brain activation'. At a behavioral level autistic children can display both hypo and hyper reactivity to changing sensory events; results from electrophysiological studies are not convergent; thus it didn't seem relevant to chose between hypo and hyper activation of the brain regions involved in change processing.

26) (2) to determine if these activations differ according to the familiarity of the stimuli

-your study is not designed to test the effects of the familiarity of the sounds on brain activation. Deviants and novels might differ in familiarity (what ever it means) but they differ also in frequency content and probability.

-Based on your introduction it seems that with deviant vs. novel contrast you are after the brain mechanism underlying involuntary attention switching (P3a). Familiarity is not an issue here.

As the difference is both in frequency spectrum and in probability, we assumed that it does lead to a difference in term of familiarity (How can a sound never heard before (novel) seem as familiar as a sound that, although rare, has been listened several times (deviant)?).

We replaced the term 'familiarity' by 'novelty' (which includes the notions of frequency content and probability). 
27) You are imaging the activation associated with auditory change detection (MMN, N1 enhancement) and involuntary control of attention (P3a). Introduction does not make a clear separation between these two concepts although this is highly relevant to your study.

This has been changed: ' We predicted that in autism we would observe a perturbation of the cerebral network involved in involuntary attention switching during auditory change detection..'.

28) "We used event-related fMRI to study frequency-deviant and complex novel sounds detection and to highlight the brain regions underpinning these processes in children with and without autism." -> Change to something like this: We used event-related fMRI to study the processing of unattended freq deviants and novel sounds ...o

This has been corrected accordingly: 'We used event-related fMRI to study the processing of unattended frequency-deviant and complex novel sounds'.

Discussion

29) "This study is the first attempt to elucidate the brain processes involved in auditory deviance and noveltydetection in autism,"

-as if different brain processes are involved in auditory deviance and novelty-detection in people with autism

-you are studying how autism affects/modulates/changes these mechanisms

'Elucidate' has been replaced by 'address' and 'autism' replaced by 'in children with autism'. But see response to comment 14 also.

30) "As predicted, the results showed that despite normal processing of infrequent acoustic changes at the level of the auditory cortex," -no such prediction presented in Introduction

The MMN topographical study we did in children with autism showed abnormal MMN distribution over the left frontal region, which was not related to any abnormality of the temporal component of the MMN. Moreover, at the end of the introduction we made prediction regarding abnormalities in frontal and parietal regions, not in the auditory areas.

\section{1) First paragraph of Discussion}

- Usually it is assumed that supratemporal (auditory cortex) mechanisms are involved in auditory change detection and other areas activated by the unattended sound changes are associated with control of attention or something of that nature. Based on Discussion, you seem to think that auditory cortex is associated with "processing of infrequent acoustic changes" and other areas outside supratemporal cortex are involved in "change detection".

'regions involved in involuntary attention switching during change detection displayed atypical activation in autism.' is now specified.

32) Our results in the typical population replicate those obtained in previous studies of auditory change detection in adults and provide new data from children.

- your results are not from "typical population" (kids), your results are consistent with previous adult studies (not replication if subjects are different and you also make the point that the activation is different in children than in adults!), the sentence says that the present results obtained in kids provide new data on kids.

The sentence has been modified:' Our results in typical children are consistent with those obtained in previous studies of auditory change detection in adults.'

33) The larger activation in the right more than in the left superior temporal gyrus during deviance detection accords with previous magnetoencephalographic (MEG) findings (Levänen et al., 1996). These results suggest that two dipoles are necessary to model MMN...

- it is strange that from discussion of your fMRI results you jump to dipole models of MMN. No discussion on dipole models of MEG data is needed here.

To our opinion, this information is relevant as it contributed to explain the larger activation we found on the right hemisphere. We added that electrophysiological data also support this idea: 'The larger 
activation in the right more than in the left temporal region during deviance detection accords with previous electrophysiological (Giard et al., 1995) and magnetoencephalographic (MEG) findings (Levänen et al., 1996)'

- in this paragraph you write (1) that the observed right hemisphere dominance is consistent with Levanen et al (you should cite other studies too), (2) that it has been suggested that planum temporale (PT) is associated with nonspecific change detection, (3) the function of PT is contrasted with that of some non-specified area which is involved in analysis of stimulus features

- I don't understand how 1 and 2 are related

-PT is not connected to your results; did you get PT activation too? Is posterior temporal cortex $=$ PT =temporoparietal junction? It is not clear which results of the present study you are comparing to those of Levanen and Mustovic.

This remark is quite confusing as we have never written the words planum temporale (PT) in this paragraph. Levänen et al found an additional generator on the right hemisphere and this was located in the inferior temporal cortex/TPJ, not in the planum temporale. We then elaborated on the potential role of this additional generator (2). And (3) we added that comparable findings have been provided using fMRI (TPJ activation to infrequent silence). Our study showed extension of the activation on the right TPJ also. In order to make it clearer, we have partially rewritten this paragraph: 'These MEG results suggest that two dipoles are necessary to model MMN activity in the right hemisphere. The additional right generator is located in the inferior parietal lobule/temporo-parietal junction and would be involved in a more global and non-specific change detection process (Levänen et al., 1996). This finding of more general processing in the temporo-parietal junction has been confirmed in an fMRI study showing that the right posterior superior temporal gyrus is activated by brief periods of silence used as deviant events, and thus plays a specific role in the integration of sequential auditory events rather than in the analysis of stimulus features (Mustovic et al., 2003). '

34) Comparison of our data with results from other studies remains difficult since there have not yet been other fMRI studies of the oddball paradigm in children.

$->$ [The conclusion of this should be: to study how the fMRI activations in kids and adults differ one has to conduct the same study in adults and in kids]

Please, read carefully, this is exactly what was written p15 at the end of the second paragraph.

35) "Thus we cannot establish clearly whether the highly significant activations found for the deviance contrast are related to the paradigm we used or to the age of the population studied."

- I do not understand this, if the activations are not related to paradigm how can they be significant (if caused by something random).

Here we refer to activation related to the fact that we used a quite 'original' paradigm (remember, the stimulus delivery was synchronized with the slice acquisition..). This might have been more efficient to elicit auditory activations. We added this information: '...the highly significant activations found for the deviance contrast are related to the original paradigm we used (stimulus delivery in the 'silent' gap)...'

36) What does "stimulus evaluation" mean? Or "detection of non-routine situations"?

ACC has been shown to have a role in modulating attention functions by influencing sensory selection. It is assumed to play a role in many cognitive tasks by allocating attentional resources when confronted with competing information processing streams. 'Non routine situations' is used in contrast with known repetitive situations that involved only pre attentional processes.

Please, see Carter et al., 2000 and Bush et al., 1999, 2000.

37) "Interestingly, this brain area", what area?

The entire paragraph is dedicated to the anterior cingulate gyrus. 'the anterior cingulate region' has been added.

38) Novelty detection processes 'are elicited' has been replaced by 'are activated' 
39) "which are unpredictable as they are new each time"

-please be more accurate, deviants are unpredictable too, the difference between deviants and novels is not in predictability (nor familiarity)

'unpredictable' has been removed.

40) You seem to think that IFG activation is associated with "an involuntary amplification mechanism" in the case of deviants but with "involuntary switching of attention" in the case of novels. This does not make any sense.

-novels are deviants with a different probability and frequency content. There is no way you can say that the functional role of IFG activation detect for both deviants and novels is different for deviants and novels.

This paragraph has been totally rewritten: 'The frontal activity associated with the triggering of an attention switch is classically localised on the right hemisphere. However, evidence of the involvement of the left inferior frontal gyrus in the mismatch process has been provided by lesions studies (Alho et al., 1994; Alain et al., 1998) and fMRI studies (Doeller et al.,2003; Rinne et al., 2005), and in the present study we also found activation in the left frontal region in the pattern common to both groups. This leads us to suggest that the left inferior frontal gyrus could also house an attention switch mechanism associated with deviance-detection. Our results suggest that such mechanism that would enhance the automatic perception of potentially relevant, deviant events is atypical in autism.'

41) "As suggested by these authors, this right dominance could be due to the frequency complexity of the sounds used for both deviant and novel stimuli (no simple sinusoidal sounds), which is more likely to be processed within the right superior temporal cortex (Wessinger et al., 2001)."

- I do not understand this sentence. Who is suggesting what? Sounds used for deviant and novel stimuli? "which is" refers to what? "right dominance" -> right hemisphere dominance. How does this explain your findings?

'These authors' refers to 'Muller et al.' 'Which is' refers to 'the frequency complexity'. We have changed the sentence in order to make it more readable.

42) "in the temporo-parietal junction. This region is assumed to be involved in the pre attentive gating mechanism" -are you making a distinction between "posterior temporal cortex" (which you connect to integration of sequential events) and "the temporo-parietal junction"? If so do you base this distinction on the data of Jaaskelainen and Mustovic? Do you think that this distinction is justified?

TPJ is involved in several cognitive processes. Moreover both a part of the inferior parietal lobule and a part of the posterior temporal cortex belong to the TPJ. We did not do the distinction between these two regions (as the studies cited didn't really allow for precise location).

43) "This suggests unusual reactivity of brain regions usually involved in change detection" -I do not understand this. What suggest unusual reactivity? what brain regions are you talking about?

This has been clarified : 'The atypical activation in the temporo-parietal junction in autism...'.

44) "as if the autistic brain became 'hermetic' (or impenetrable) to novelty in one sensory modality while involved in a processing in another modality"

-this is very speculative, you do not know what the subjects are doing in the scanner. It is equally possible that the autistic kids are actively listening to the sounds and while their brain detects the sound changes, no switching of attention from the video to the sounds is needed (as they are listening to the sounds).

This is an interesting alternative. However, if autistic children were attending the sounds, one can assume that we should also have evidenced an enhancement of the activation in the (auditory) temporal region in autism as compared to controls. This wasn't observed.

45) "The current results suggest people with autism may have some difficulty in applying preattentive processes to unexpected infrequent stimuli."

- your data shows nothing like this: (1) there is no difference in the auditory cortex activation between the groups, i.e., no difference in preattentive auditory change detection, (2) this conclusion does not necessarily follow from the result that people with autism show reduced activation (in some areas). Rather it is possible that the differences the groups are caused by differences in the task performance. This is a serious methodological problem in this kind of 
clinical studies. Note that is often assumed that MMN can be used as a clinical tool as MMN is not (too much) affected by attention and vigilance. However, when studying control of attention in fMRI you are out of the 'safe' MMN world. Further, the group differences seem to suggest that autism affects involuntary control of attention rather than "applying preattentive processes to unexpected infrequent stimuli" (what ever that is supposed to mean; how can one apply processes to stimuli?).

There is a misunderstanding here. Please, note that we consider that 'the involuntary attention switch' is also a 'preattentive' process. The sentence has been replaced by: 'may have some difficulty in involuntary attention switching when processing unexpected infrequent stimuli.'

46) Your discussion goes too far away from the data (e.g., the paragraph about Mesulam's novelty seeking view). You should rewrite the discussion in the spirit of your first sentence.

We are aware that this paragraph is not fully related to our study. However, in the text we carefully mentioned that this is a 'framework for discussing' our findings. Moreover, we do think that reading the 'repetitive and restrictive' aspect of autism in the perspective developed by Mesulam is interesting regarding the autistic pathology as it allows making a bridge between cognitive style and clinics.

47) You discuss the potential differences between autistic and normal kids in the video watching task. You seem to think that even if the autistic kids are doing a different task than the normal kids, the results would still reveal something interesting about the abnormal brain mechanisms underlying autism. This is not necessarily the case. It is possible that the differences between the two groups are only caused by the differences in the tasks the kids are performing. This is a serious problem. Please do not try to hide it. Discuss it. Think what could be done to control for it.

We added: 'Controlling for such attentional effects may be possible if a concurrent visual task was presented to the participants while the sound sequence is delivered.'

(not sure this would be possible with the autistic kids though..).

48) "To summarise, our study found atypical activation of brain regions involved in the automatic detection of changing auditory events in children with autism."

-this seems wrong: your results show clearly that there were absolutely no differences between the groups in auditory change detection (auditory cortex). However, you found that Dev $>$ Sta contrast revealed differences in cingulate and frontal lobes. It seems that these areas are more likely to be associated with involuntary control of attention or something like that rather than auditory change detection. That is, it seems that the group differences are more like to be related to control of attention rather that change detection. This seems to fit with your idea of autism.

The sentence has been changed: 'To summarize, our study found atypical activation of brain regions involved in involuntary attention switching associated with automatic detection of changing auditory events in children with autism.

Please note that the abnormalities found here regarding the control of attention are related to change perception only. We might not be authorized to extrapolate this group difference to any other kind of attentional control. 


\section{Reviewer 3:}

1 - As suggested, the statistical analysis section has been improved (p11 first paragraph).

'Differences between groups were evaluated in a similar manner by computing for each contrast the SPM $\{F\}$ statistics thresholded at $P<0.001$ uncorrected. Thus, the only difference between the two analyses (group as a whole and between groups comparison) was in the FDR correction which was applied for the population effects only.'

2 - We added in the manuscript a short paragraph regarding the laterality of the prefrontal activity and the evidence of left prefrontal involvement in preattentive deviance processing:

'Additionally, the current data indicate lower activation in the left inferior frontal gyrus in the autism group during deviance-detection. The frontal activity associated with the triggering of an attention switch is classically localised on the right hemisphere. However, evidence of the involvement of the left inferior frontal gyrus in the mismatch process has been provided by lesions studies (Alho et al., 1994; Alain et al., 1998) and fMRI studies (Doeller et al.,2003; Rinne et al., 2005), and in the present study we also found activation in the left frontal region in the pattern common to both groups. This leads us to suggest that the left inferior frontal gyrus could also house an attention switch mechanism associated with deviancedetection. Our results suggest that such mechanism that would enhance the automatic perception of potentially relevant, deviant events is atypical in autism.' 
Figure
Click here to download high resolution image

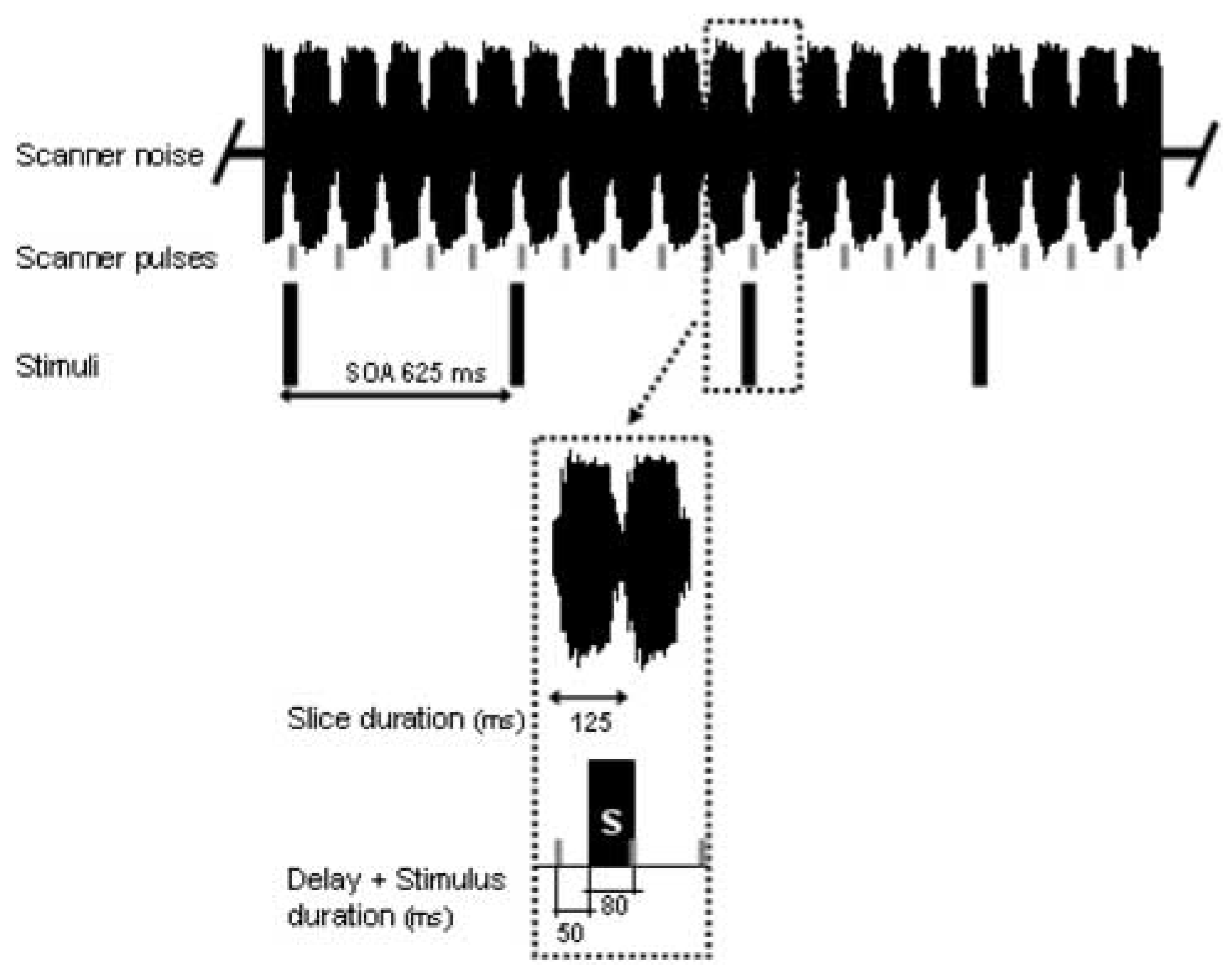


Figure
Click here to download high resolution image

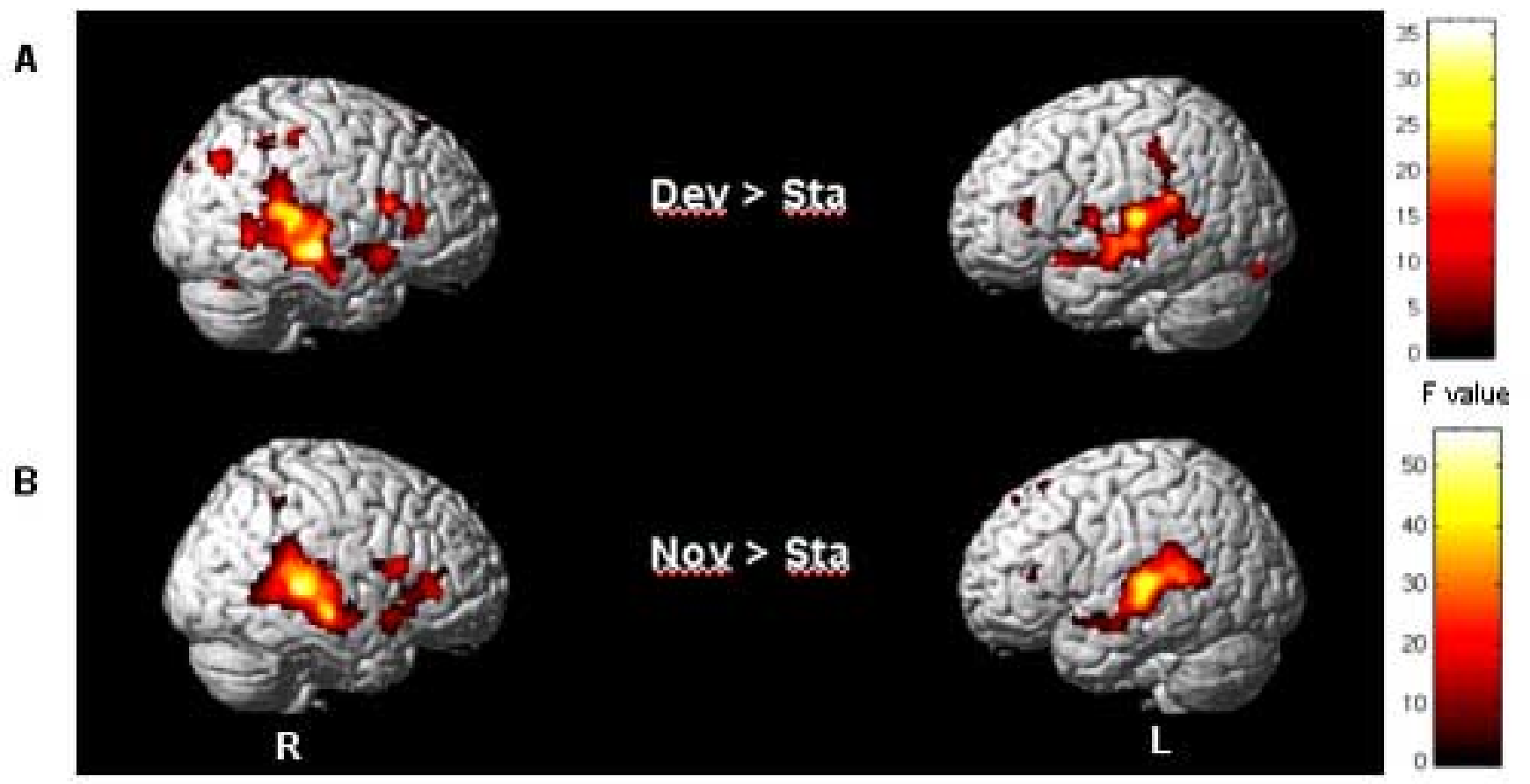


Click here to download high resolution image

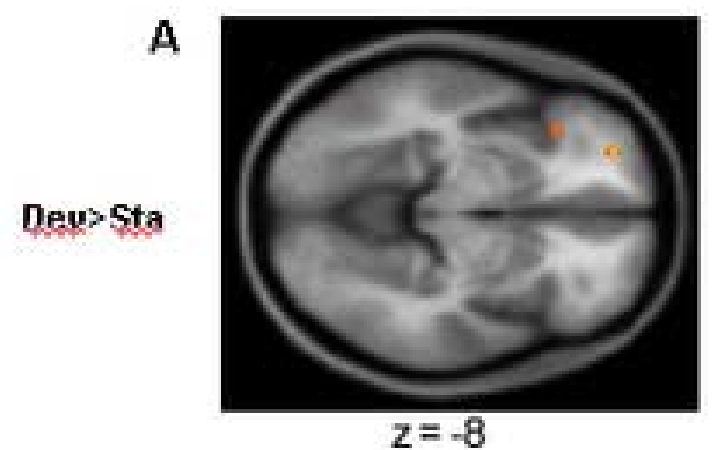

$z=-8$

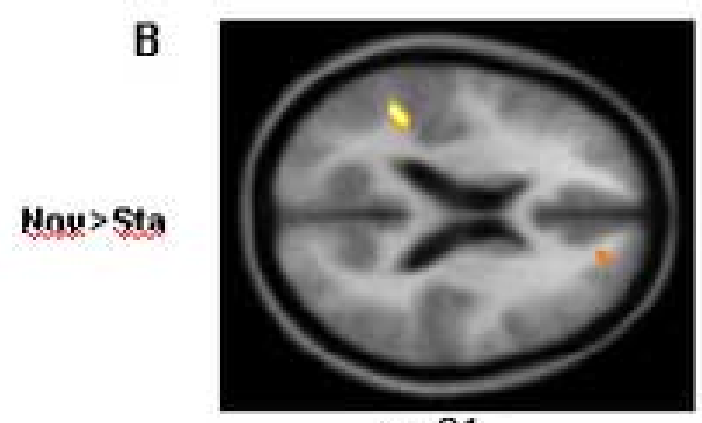

$z=21$

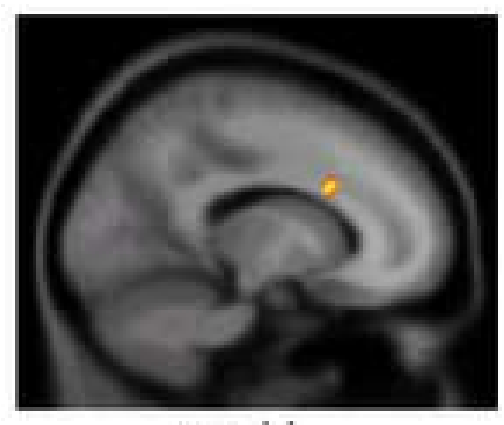

$x=-14$

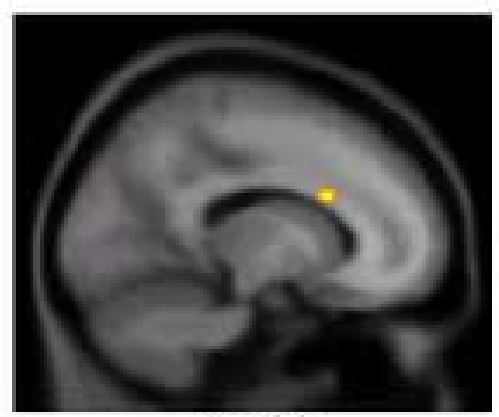

$x=-14$
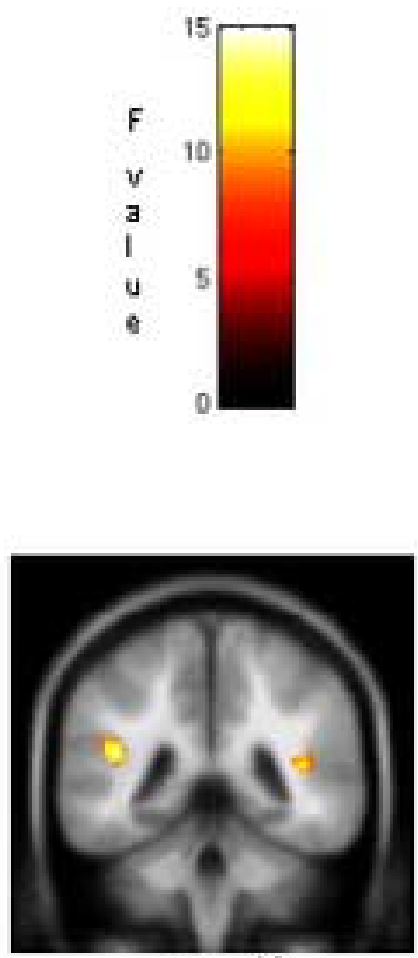

$y=-40$ 
Click here to download high resolution image

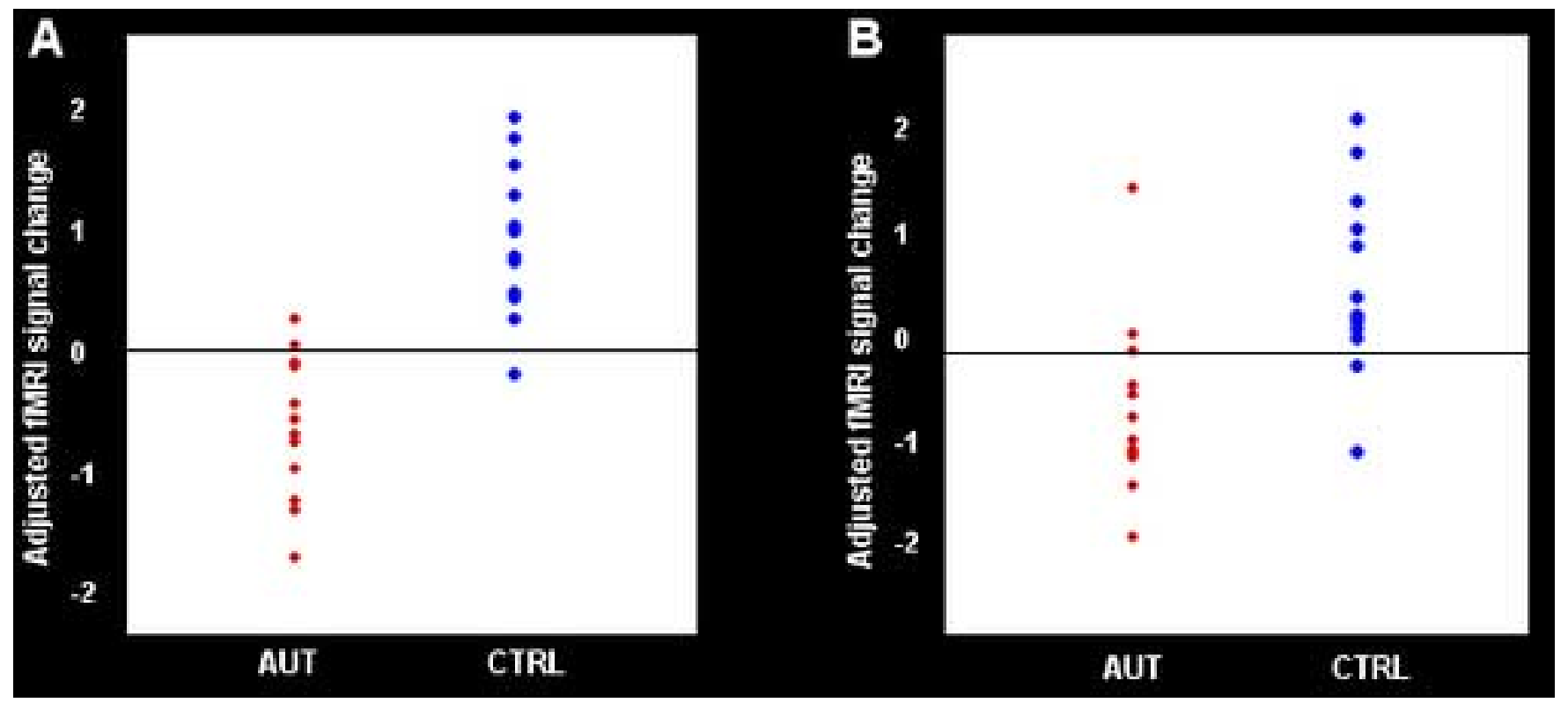




\begin{tabular}{|c|c|c|c|c|c|}
\hline $\begin{array}{l}\text { Functional } \\
\text { comparison }\end{array}$ & $\begin{array}{c}\text { Cluster } \\
\text { size } \\
\text { (voxels) }\end{array}$ & Brain regions & $\mathbf{B A}$ & $\begin{array}{c}\text { Talair ach } \\
\text { coordinates } \\
(x, y, z)\end{array}$ & Zscore \\
\hline \multirow[t]{16}{*}{ Dev $>$ Sta } & 3040 & R Mid Temporal Gyrus & 21 & $57,-22,-6$ & 6.17 \\
\hline & & R Sup Temporal Gyrus & 22 & $47,-33,7$ & 5.89 \\
\hline & & R Temporo Parietal Junction & 40 & $61,-34,20$ & 4.37 \\
\hline & 2272 & L Superior Temporal Gyrus & 22 & $-63,-23,7$ & 5.30 \\
\hline & & L Mid Temporal Gyrus & 21 & $-48,-16,-9$ & 4.87 \\
\hline & & L Inf Parietal Lobule & 40 & $-46,-37,35$ & 4.65 \\
\hline & 800 & R Cingulate & 23 & $3,-28,27$ & 4.87 \\
\hline & & R Medial Dorsal Thalamus & NA & $6,-13,8$ & 4.12 \\
\hline & & R Head of Caudate & NA & $18,-5, \quad 13$ & 4.05 \\
\hline & 376 & R Inf Parietal Lobule & 7 & $4,-60,47$ & 4.42 \\
\hline & 287 & R Inf Frontal Gyrus & 44 & $57,16,12$ & 4.60 \\
\hline & 231 & R Inf Parietal Lobule & 40 & $38,-68,37$ & 4.72 \\
\hline & 218 & R Medial Temporal Lobe & 34 & $18, \quad 1,-15$ & 4.36 \\
\hline & 82 & R Cerebellum & NA & $34,-65,-20$ & 4.51 \\
\hline & 55 & L Cerebellum & NA & $-28,-88,-12$ & 4.60 \\
\hline & 53 & L Inf Frontal Gyrus & 45 & $-51,33,9$ & 4.03 \\
\hline \multirow[t]{6}{*}{ Nov $>$ Sta } & 3209 & R Mid Temporal Gyrus & 21 & $57,-20,-6$ & 7.13 \\
\hline & & R Sup Temporal Gyrus & 41 & $42,-36,11$ & 6.53 \\
\hline & & R Temporo Parietal Junction/ & $40 / 22$ & $65,-38,22$ & 5.61 \\
\hline & & Sup Temporal Gyrus & & & \\
\hline & 2582 & L Sup Temporal Gyrus & 22 & $-63,-27,7$ & 6.22 \\
\hline & & L Sup Temporal Gyrus & 22 & $-38,-38,18$ & 5.41 \\
\hline
\end{tabular}




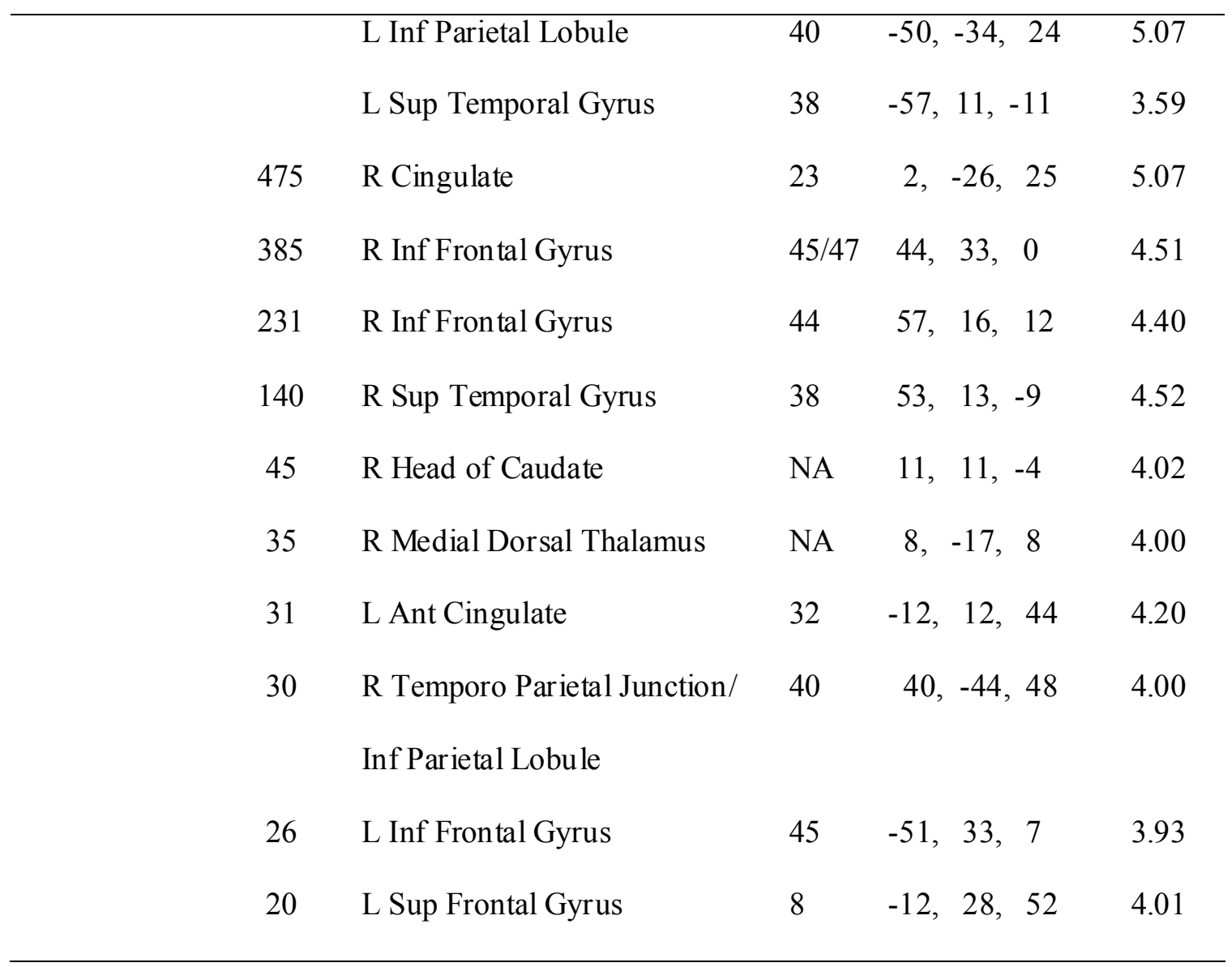

Table 1: Activation common to both groups $(\mathrm{P}<0.01$, FDR corrected $)$ 


\begin{tabular}{|c|c|c|c|c|c|}
\hline $\begin{array}{l}\text { Functional } \\
\text { comparison }\end{array}$ & $\begin{array}{c}\text { Cluster } \\
\text { size } \\
\text { (voxels) }\end{array}$ & Brain region & $\mathbf{B A}$ & $\begin{array}{l}\text { Talairach } \\
\text { coordinates } \\
(x, y, z)\end{array}$ & Z score \\
\hline \multirow[t]{3}{*}{ Dev $>$ Sta } & 40 & L Ant Cingulate & $24 / 32$ & $-13,19, \quad 23$ & 4.25 \\
\hline & 18 & L Mid Orbito-Frontal Gyrus & 11 & $-26,50,-11$ & 3.74 \\
\hline & 16 & L Inf Frontal Gyrus & $47 / 11$ & $-31,25,-3$ & 3.27 \\
\hline \multirow[t]{7}{*}{ Nov $>$ Sta } & 91 & L Inf Parietal Lobule/ & $40 / 22$ & $-43,-42,20$ & 4.11 \\
\hline & & Temporo parietal junction & & & \\
\hline & 42 & R Cerebellum & NA & $6,-49,-16$ & 3.93 \\
\hline & 33 & R Sup Temporal Gyrus & 22 & $42,-40,13$ & 3.54 \\
\hline & 24 & R Inf Frontal Gyrus & 47 & $28,32,-20$ & 3.71 \\
\hline & 20 & L Ant Cingulate & 24 & $-15,17,23$ & 3.62 \\
\hline & 20 & R Mid Frontal Gyrus & 9 & $20,50,18$ & 3.54 \\
\hline
\end{tabular}

Table 2: Direct groups comparison Control $>$ Autism $(\mathbf{P}<0.001$, uncorrected $)$ 